Notfall Rettungsmed 2015 $\cdot 18: 964-983$ DOI 10.1007/s10049-015-0090-0

Online publiziert: 9. November 2015

(C) European Resuscitation Council (ERC),

German Resuscitation Council (GRC), Austrian

Resuscitation Council (ARC) 2015

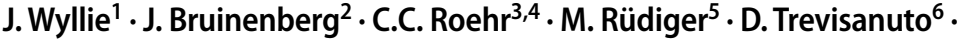
B. Urlesberger ${ }^{7}$

${ }^{1}$ Abteilung für Neonatologie, The James Cook University Hospital, Middlesbrough, Großbritannien

${ }^{2}$ Abteilung für Kinderheilkunde, Sint Elisabeth Hospital, Tilburg, Niederlande

${ }^{3}$ Abteilung für Neonatologie, Charité Universitätsmedizin, Berlin, Deutschland

${ }^{4}$ Newborn Services, John Radcliffe Hospital, Oxford University Hospitals, Oxford, Großbritannien

${ }^{5}$ Abteilung für Neonatologie, Medizinische Fakultät Carl Gustav Carus, TU Dresden, Dresden, Deutschland

${ }^{6}$ Abteilung für Frauen- und Kindergesundheit, Padua University, Azienda Ospediliera di Padova, Padua, Italien

${ }^{7}$ Abteilung für Neonatologie, Medizinische Universität Graz, Graz, Österreich

\title{
Die Versorgung und Reanimation des Neugeborenen
}

\author{
Kapitel 7 der Leitlinien zur Reanimation \\ 2015 des European Resuscitation Council
}

\section{Einleitung}

Die folgenden Leitlinien zur Neugeborenenreanimation sind das Ergebnis des „2015 International Consensus on Cardiopulmonary Resuscitation and Emergency Cardiovascular Care Science with Treatment Recommendations (CoSTR 2015)“ $[1,2]$. Sie stellen eine Aktualisierung der bereits durch das ERC veröffentlichten Leitlinien [3] dar und berücksichtigen Empfehlungen nationaler sowie internationaler Organisationen und bestehende evidenzbasierte Erkenntnisse [4].

\section{Zusammenfassung der Veränderungen im Vergleich zu den Leitlinien 2010}

Die neuen Leitlinien 2015 zur Stabilisierung und Reanimation des Neugeborenen beinhalten folgende wesentliche Veränderungen:

- Unterstützung der Anpassung: Die Situation nach der Geburt ist einzigartig im Leben. Neugeborene benötigen selten eine vollständige Re-

Die Autoren der Leitlinien gedenken Sam Richmond und danken ihm für seine wertvollen Beiträge zu diesem Kapitel. animation, aber mitunter stabilisierende Maßnahmen. Der Terminus „Unterstützung der Anpassung“ wurde zur besseren Unterscheidung zwischen Reanimationsmaßnahmen, die Organfunktionen wiederherstellen sollen, und unterstützenden Maßnahmen während der Umstellung des Körpers eingeführt.

- Abnabeln: Für unbeeinträchtigte, gesunde Neugeborene wird ein verzögertes Abnabeln, frühestens 1 min nach der Geburt, empfohlen. Dies gilt für reife Neugeborene und Frühgeborene. Für Neugeborene, die Reanimationsmaßnahmen benötigen, können derzeit aufgrund fehlender Daten keine Empfehlungen bezüglich des idealen Zeitpunkts des Abnabelns gegeben werden.

- Temperatur: Die Körpertemperatur von nicht asphyktischen Neugeborenen soll zwischen 36,5 und $37,5^{\circ} \mathrm{C}$ gehalten werden. Da das Wärmemanagement für gesunde Neugeborene einen großen Einfluss auf Morbidität und Mortalität hat, soll in diesen Leitlinien nochmals besonders darauf hingewiesen werden. Die Körpertemperatur bei Aufnahme soll immer dokumentiert und als Prädiktor für das
Outcome und als Qualitätsmerkmal der Versorgung angesehen werden.

- Wärmemanagement bei Frühgeborenen: Bei Frühgeborenen < 32 Schwangerschaftswochen ist eine Kombination von mehreren Maßnahmen notwendig, um nach der Aufnahme und während der Stabilisierung eine Temperatur von 36,5 bis $37,5^{\circ} \mathrm{C}$ zu erreichen und aufrechtzuerhalten. Dies kann gewärmte und befeuchtete Atemgase, eine Erhöhung der Raumtemperatur und zusätzlich das Einwickeln von Körper und Kopf (unter Aussparung des Gesichtes) in eine Plastikfolie und/oder eine Versorgung auf einer Wärmematte beinhalten. Alle diese Maßnahmen können eine Hypothermie verhindern.

- Optimale Bestimmung der Herzfrequenz: Bei Neugeborenen, die Reanimationsmaßnahmen benötigen, wird angeregt, ein EKG zur schnellen und sicheren Bestimmung der Herzfrequenz zu verwenden.

- Mekonium: Die tracheale Intubation eines avitalen Neugeborenen mit Mekonium soll nicht mehr routinemäßig, sondern nur noch bei Verdacht auf eine Obstruktion der Trachea durchgeführt werden. Entscheidend ist, bei fehlender oder insuffi- 


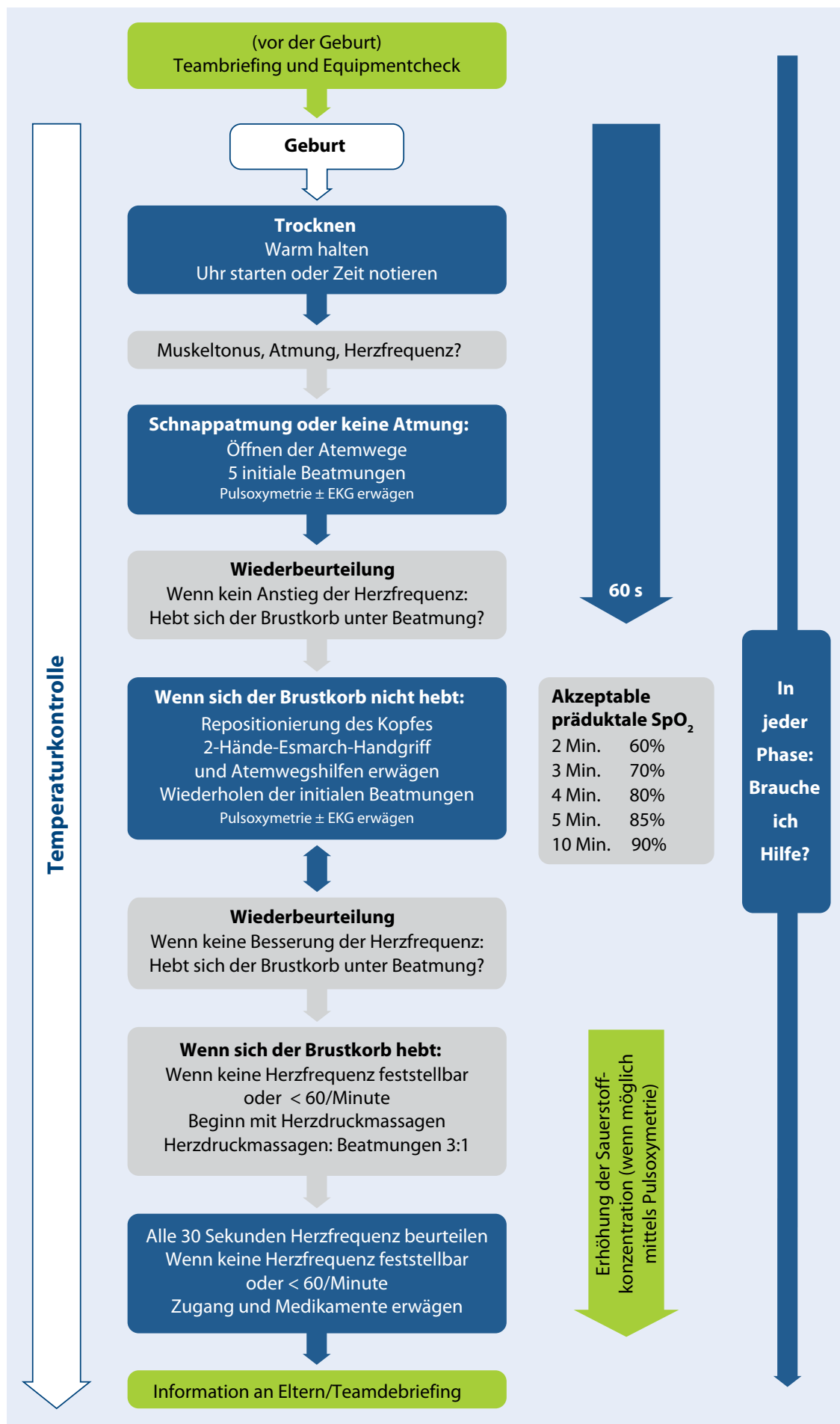

Abb. $1 \Delta$ Algorithmus der Neugeborenenreanimation

zienter Spontanatmung innerhalb der ersten Lebensminute mit einer Beatmung zu beginnen und dies nicht zu verzögern.

- Raumluft/Sauerstoff: Die Beatmung eines reifen Neugeborenen soll mit Raumluft beginnen. Für Frühgebore- ne kann anfangs ebenfalls Raumluft oder eine geringe Sauerstoffkonzentration (bis $30 \%$ ) verwendet werden. Wenn es trotz effektiver Beatmungen zu keinem zufriedenstellenden Anstieg der Sauerstoffkonzentration kommt (idealerweise gemessen über eine Pulsoxymetrie), soll eine Erhöhung der Sauerstoffkonzentration in Erwägung gezogen werden.

- CPAP: Für ein spontan atmendes Neugeborenes mit Zeichen einer angestrengten Atmung hat eine Atemunterstützung mittels CPAP einen höheren Stellenwert als eine Intubation.

Die vorliegenden Leitlinien definieren sicher nicht den einzig gangbaren Weg für die Reanimation eines Neugeborenen. Vielmehr entsprechen sie einer weit verbreiteten und akzeptierten Auffassung über eine sichere und effektive Durchführung von Reanimationsmaßnahmen nach der Geburt (• Abb. 1).

\section{Vorbereitung}

Die Anpassung vom fetalen Leben an das Leben nach der Geburt erfordert anatomische und physiologische Veränderungen. Mit dem Ende des intrauterinen Gasaustausches über die Plazenta müssen sich die intrauterin mit Flüssigkeit gefüllten Lungen mit Luft füllen, um den pulmonalen Gasaustausch zu übernehmen. Die Absorption von Fruchtwasser, die Füllung der Lungen mit Luft, die ersten Atemzüge und das Ende der plazentaren Versorgung sind die wesentlichen Veränderungen in dieser Phase.

Nur eine sehr geringe Anzahl von Neugeborenen benötigt eine Reanimation nach der Geburt. Allerdings brauchen einige Neugeborene in der oben beschriebenen Anpassungsphase unterstützende Maßnahmen. Werden diese nicht durchgeführt, können als Folge letztlich doch Reanimationsmaßnahmen erforderlich werden. Meistens bestehen diese dann jedoch lediglich in einer kurzen assistierten Belüftung der Lungen. Nur eine kleine Minderheit braucht zusätzlich zur Belüftung der Lungen kurzzeitig Thoraxkompressionen. In einer retrospektiven Studie zeigten $85 \%$ der reifen Neugeborenen innerhalb von 10-30 s nach der Geburt eine Spontanatmung, weitere $10 \%$ begannen unter Abtrocknen und Stimulation zu atmen, bei etwa $3 \%$ setzte eine Spontanatmung letztlich unter Maskenventilation ein. Lediglich $2 \%$ mussten zur Atemunterstützung intubiert werden, und bei nur $0,1 \%$ waren Thoraxkompres- 
sionen und/oder eine Adrenalingabe notwendig [5-7]. Von 97.648 in einem Jahr in Schweden geborenen Babys mit einem Gewicht von über $2500 \mathrm{~g}$ waren bei lediglich 10 von 1000 (1\%) postnatale Reanimationsmaßnahmen notwendig [8]. In 8 von 1000 Fällen war eine Maskenbeatmung ausreichend, lediglich 2 von 1000 Neugeborenen mussten intubiert werden. Dieselbe Studie untersuchte die Häufigkeit unvorhergesehener Reanimationen nach der Geburt. Bei Neugeborenen mit geringem Risiko (problemlose Entbindung nach der 32. Schwangerschaftswoche) waren lediglich in 2 von 1000 Fällen $(0,2 \%)$ unterstützende bzw. Reanimationsmaßnahmen notwendig. Von diesen $0,2 \%$ sprachen $90 \%$ auf eine alleinige Maskenbeatmung an, die übrigen $10 \%$ stabilisierten sich nicht unter Maskenbeatmung und wurden daher intubiert. Thoraxkompressionen waren nahezu niemals notwendig.

Das Risiko für Reanimationsmaßnahmen oder stabilisierende Maßnahmen nach der Geburt ist allerdings höher bei einem bereits peripartalen Hinweis auf eine schwerwiegende Beeinträchtigung des Fetus, bei Frühgeborenen unter der 35. Schwangerschaftswoche, bei Zangengeburten, mütterlichen Infektionen oder Mehrlingsschwangerschaften [9]. Neugeborene nach einer Entbindung per Sectio caesarea, v. a. vor der 39. Schwangerschaftswoche, haben ebenfalls ein höheres Risiko für respiratorische Anpassungsstörungen und benötigen häufiger unterstützende Maßnahmen [10-13]. Elektive Schnittentbindungen am Geburtstermin zeigen bei Fehlen weiterer Risikofaktoren allerdings keine erhöhte Wahrscheinlichkeit für Reanimationsmaßnahmen [14-17].

Da es zwar häufig, aber nicht immer möglich ist, die Notwendigkeit von Reanimationsmaßnahmen vorherzusehen, und prinzipiell jedes Neugeborene während und nach der Geburt Unterstützung benötigen kann, soll bei jeder Geburt in Neugeborenenreanimation trainiertes Personal schnell und leicht verfügbar sein. Bei Risikogeburten muss darüber hinaus speziell neonatologisch ausgebildetes und trainiertes Personal zur Verfügung stehen, und zumindest eine Person muss in der Intubation von Neugeborenen er- fahren sein. Ist die Reanimation des Neugeborenen notwendig, muss dies die einzige und ausschließliche Aufgabe dieses Teams sein. Basierend auf aktueller Praxis und klinischer Qualitätsprüfung sollen lokale Leitlinien entwickelt werden, die festlegen, welches Personal bei Geburten anwesend sein soll. Jede Institution muss über ein Notfallprotokoll verfügen, das die Alarmierungsstruktur klar regelt und eine schnelle Verfügbarkeit von in Neugeborenenreanimation ausgebildetem und trainiertem Personal zu jeder Zeit ermöglicht. Wann immer die Zeit es erlaubt, soll das versorgende Team zunächst ein Briefing erhalten, und die Rollen im Team sollen eindeutig verteilt werden. Ebenso ist es wichtig, die Eltern auf eine möglicherweise notwendig werdende Reanimation vorzubereiten.

Ein strukturiertes Ausbildungsprogramm für Standards und Fertigkeiten der Neugeborenenreanimation ist daher für jede geburtshilflich tätige Einrichtung unabdingbar. Kontinuierliches Lernen und Praktizieren sind notwendig, um die klinischen Fertigkeiten zu erhalten.

\section{Geplante Hausgeburten}

Von Land zu Land finden sich unterschiedliche Empfehlungen, welche Personen bei einer geplanten Hausgeburt anwesend sein sollen. Ist die Entscheidung für eine geplante Hausgeburt in Abstimmung mit dem Arzt und der Hebamme gefallen, müssen auch hierbei die Standards der Neugeborenenversorgung bezüglich initialer Beurteilung des Neugeborenen, stabilisierender Maßnahmen und einer mitunter notwendigen Reanimation gelten. Bereits bei der Planung einer Hausgeburt muss die werdende Mutter darüber aufgeklärt werden, dass aufgrund der schwierigeren Verfügbarkeit weiterer Hilfe eine Reanimation in häuslicher Umgebung zwangsläufig nicht im vollen Umfang durchgeführt werden kann. Idealerweise sollen bei allen Hausgeburten zwei trainierte professionelle Helfer anwesend sein. Mindestens einer der beiden Helfer muss in der Durchführung von Maskenbeatmung und Thoraxkompressionen bei Neugeborenen gut trainiert und erfahren sein.

\section{Material und Vorbereitung}

Im Gegensatz zu einer Reanimation im Erwachsenenalter ist eine Reanimation nach der Geburt oft ein vorhersehbares Ereignis. Daher ist es meist möglich, Umgebung und Ausrüstung noch vor der Entbindung eines Babys entsprechend vorzubereiten. Die Versorgung eines kritisch kranken Neugeborenen soll in einer warmen, gut beleuchteten und zugluftfreien Umgebung stattfinden. Das Baby soll unter einem Heizstrahler (im klinischen Umfeld) auf eine gerade und glatte Fläche gelegt werden. Sämtliches zur Reanimation notwendige Material muss leicht verfügbar sein. Es muss regelmäßig auf Vollständigkeit und Funktion überprüft werden.

Findet eine Geburt außerhalb der üblichen Entbindungsbereiche statt, soll als Mindestausstattung folgendes Equipment zur Verfügung stehen:

- ein Hilfsmittel zur sicheren, assistierten Beatmung in der passenden Größe für Neugeborene,

- warme, trockene Tücher und Laken,

- sterile Instrumente zum Abklemmen und Durchtrennen der Nabelschnur,

- saubere Handschuhe für alle Versorgenden.

Da unerwartete Geburten außerhalb des klinischen Umfelds vor allem das Personal des Rettungsdienstes betreffen, soll dieses auf die Versorgung von Neugeborenen vorbereitet und trainiert sein.

\section{Abnabelungszeitpunkt}

Neugeborene, deren Nabelschnur vor dem ersten Atemzug abgeklemmt wurde, zeigen in cineradiographischen Studien während der ersten Atemzüge nach der Geburt eine sofortige Verminderung der Herzgröße für die folgenden 3 bis 4 Herzzyklen. Danach wird das Herz wieder größer, etwa entsprechend der Größe des fetalen Herzens. Die initiale Größenabnahme erklärt sich durch eine Verringerung des Lungengefäßwiderstands nach Füllung des nun durchbluteten pulmonalen Gefäßsystems durch die Öffnung der Lungen. Die anschließend zu beobachtende Größenzunahme lässt sich demnach durch das nun von der Lunge zum Her- 
zen wieder zurückfließende Blut interpretieren [18]. Brady et al. beschrieben eine Bradykardie bei einem Abklemmen der Nabelschnur vor dem ersten Atemzug. Diese ließ sich bei einem Abklemmen der Nabelschnur erst nach Etablierung der Atmung nicht nachweisen [19]. Entsprechend einer tierexperimentellen Studie mit Lämmern scheint diese Beobachtung auch für Frühgeborene zu gelten [20].

Ein spätes Abnabeln hat in Studien eine Verbesserung des Eisenstatus und weiterer Werte des roten Blutbilds in den ersten 3 bis 6 Monaten postnatal gezeigt. Frühgeborene wiesen eine geringere Transfusionsbedürftigkeit auf [21, 22]. Diese Studien beschreiben ebenfalls bei den spät abgenabelten Kindern die häufigere Notwendigkeit einer Phototherapie im Rahmen einer Hyperbilirubinämie. Das konnte in einer randomisiert-kontrollierten Studie allerdings nicht nachgewiesen werden [21].

Eine systematische Übersicht zum späten Abnabeln und Ausstreifen der Nabelschnur („umbilical cord milking“) bei Frühgeborenen zeigte im Vergleich zur Kontrollgruppe eine verbesserte klinische Stabilisierung in der direkten postnatalen Phase sowie einen höheren mittleren Blutdruck (MAD) und höhere Hämoglobinwerte bei Aufnahme [23]. Außerdem waren bei diesen Kindern in den folgenden Wochen weniger Bluttransfusionen notwendig [23]. Einige Studien scheinen zudem für eine geringere Inzidenz an intraventrikulären Blutungen, dem Auftreten einer Periventrikulären Leukomalazie [22, 24, 25] und Late-onset-Septitiden [24] zu sprechen.

Es gibt bisher keine Humandaten, die den Einfluss eines späten Abnabelns bei reanimationspflichtigen Neugeborenen beschreiben, da diese Kinder aus den entsprechenden Studien immer ausgeschlossen wurden.

Unbeeinträchtigte Neugeborene, die keine Reanimationsmaßnahmen benötigen, sollen daher verzögert, frühestens nach $1 \mathrm{~min}$, abgenabelt werden. Diese Empfehlung gilt auch für stabile Frühgeborene. Bis zur Verfügbarkeit neuer Erkenntnisse sollen Neugeborene, die nicht atmen oder schreien, sofort abgenabelt werden, damit unverzüglich mit effektiven Reanimationsmaßnahmen begonnen werden kann. Ein Ausstreifen der Nabelschnur nach dem Abnabeln könnte möglicherweise bei diesen Kindern eine sinnvolle Alternative sein, allerdings besteht bisher zu wenig Evidenz, um diese Maßnahme routinemäßig empfehlen zu können $[1,2]$. Das Ausstreifen der Nabelschnur im Rahmen einer Sectio caesarea führt zu einer verbesserten hämatologischen Situation in der direkten postnatalen Phase, einer höheren Körpertemperatur bei Aufnahme und einer erhöhten Urinproduktion im Vergleich zu einem verzögerten Abnabeln (>30 s). Bei spontangeborenen Neugeborenen konnte dies allerdings nicht beobachtet werden [26].

\section{Wärmemanagement}

Nackte, feuchte Neugeborene sind in einem Raum, der für Erwachsene angenehm warm erscheint, nicht in der Lage, ihre Körpertemperatur zu halten.

Beeinträchtigte Neugeborene sind bezüglich eines Wärmeverlusts besonders empfindlich [27]. Kälte bedeutet Stress für ein Neugeborenes und führt zu einer verminderten arteriellen Sauerstoffkonzentration [28] sowie einer zunehmenden metabolischen Azidose [29].

Der Zusammenhang zwischen Hypothermie und Mortalität ist seit mehr als einem Jahrhundert bekannt [30]. Dabei ist die Temperatur bei Aufnahme generell ein bedeutender Prädiktor der Mortalität von nicht asphyktischen Neugeborenen jeder Schwangerschaftswoche [31-65]. Frühgeborene sind durch eine Hypothermie besonders gefährdet. Folgen können schwere Komplikationen wie intraventrikuläre Blutungen [35, 42, 55, 66-69], die Notwendigkeit einer Atemunterstützung [31, 35, 37, 66, 70-74] und Hypoglykämien [31, $49,60,74-79]$ sein. Einige Studien zeigen ebenfalls eine erhöhte Inzidenz von Lateonset-Septitiden [49].

Die Temperatur von nicht asphyktischen Neugeborenen soll daher zwischen 36,5 und $37,5^{\circ} \mathrm{C}$ gehalten werden. Jedes Grad, um das diese Temperatur bei Aufnahme unterschritten wird, bedeutet eine Zunahme der Mortalität um $28 \%$ [1, 2, 49]. Die Körpertemperatur bei Aufnahme soll immer dokumentiert werden. Sie ist ein Prädiktor für das Outcome und ein Qualitätsmarker für die Versorgung.
Einem Wärmeverlust soll folgendermaßen vorgebeugt werden:

- Neugeborene sollen vor Zugluft geschützt werden [80]. Fenster müssen geschlossen und Klimaanlagen adäquat eingestellt sein [52].

- Das reife Neugeborene wird direkt nach der Geburt sorgfältig abgetrocknet. Um weiteren Wärmeverlust zu vermeiden, werden Kopf und Körper des Neugeborenen, unter Aussparung des Gesichts, mit einem warmen Tuch bedeckt. Alternativ kann das nackte Neugeborene der Mutter auf die Brust gelegt werden, und beide werden mit einem Tuch zugedeckt.

- Der Versorgungsraum soll eine Temperatur zwischen 23 und $25^{\circ} \mathrm{C}$ haben $[1,2,48,80]$. Für die Versorgung von Frühgeborenen $<28$ Schwangerschaftswochen sollte die Raumtemperatur $>25^{\circ} \mathrm{C}$ liegen $[27,48,79,81]$.

- Sind in der Anpassungsphase unterstützende oder Reanimationsmaßnahmen notwendig, wird das Neugeborene unter einem vorgewärmten Heizstrahler auf einer warmen, ebenen Fläche platziert.

- Frühgeborene unter der 32. Schwangerschaftswoche sollen unter Aussparen des Gesichts komplett in eine durchsichtige Plastikfolie gehüllt werden. Dabei wird das Kind vorher nicht abgetrocknet und so eingehüllt unter einem Wärmestrahler platziert [73, 77, 82, 83].

- Bei Frühgeborenen <32. Schwangerschaftswochen ist eine Kombination aus mehreren Maßnahmen notwendig, um während der Aufnahme und Stabilisierung eine Temperatur von $36,5-37,5^{\circ} \mathrm{C}$ zu erreichen und zu erhalten. Diese Maßnahmen können gewärmte und befeuchtete Atemgase sein, [84, 85] eine Erhöhung der Raumtemperatur in Kombination mit einer zusätzlichen Kopfbedeckung und einer Wärmematte [70, 72, $86,87]$ bzw. die alleinige Verwendung einer Wärmematte [88-92]. Alle diese Maßnahmen sollen eine Hypothermie verhindern.

- Für Neugeborene, die außerhalb der üblichen Entbindungsbereiche geboren werden, ist es möglicherweise sinnvoll, sie nach dem Trocknen 
zunächst in eine Plastikfolie zu hüllen und diese dann mit Stoffwindeln zu umwickeln [93, 94]. Alternativ können gesunde Neugeborene $>30$. Schwangerschaftswochen nach dem Trocknen zugedeckt der Mutter nackt auf die Brust gelegt werden, um die Temperatur während des Transports zu halten [95-101]. (Anmerkung der Übersetzer: In den deutschsprachigen Ländern ist ein Transport des Kindes auf dem Arm der Mutter aus versicherungsrechtlichen Gründen nicht üblich.)

Dem Schutz vor Auskühlung kommt eine besondere Bedeutung zu. Allerdings sollte auch darauf geachtet werden, eine Hyperthermie $\left(>38^{\circ} \mathrm{C}\right) \mathrm{zu}$ vermeiden. Neugeborene fiebernder Mütter haben z. B. eine höhere Wahrscheinlichkeit für ein Atemnotsyndrom, neonatale Krampfanfälle oder Zerebralparesen und eine höhere frühe Mortalität [102, 103]. In tierexperimentellen Studien konnte zudem gezeigt werden, dass eine Hyperthermie während oder nach einer Ischämie zur Vergrößerung eines Hirnschadens führt [104, 105].

\section{Initiale Beurteilung}

Der APGAR-Score war nie als Hilfsmittel gedacht, um durch die Addition von Zahlenwerten einzelner klinischer Parameter reanimationspflichtige Neugeborene zu identifizieren [106, 107]. Das rasche, simultane Erfassen einzelner Parameter des APGAR-Scores, wie Atemfrequenz, Herzfrequenz und Muskeltonus, ist jedoch hilfreich, um schnell eine Reanimationspflichtigkeit zu erkennen.

Die wiederholte Erhebung der Herzfrequenz, mehr noch als die Beurteilung der Atmung, ist ein guter Parameter, um zu beurteilen, ob sich der Zustand eines Neugeborenen unter den durchgeführten Maßnahmen bessert oder weitere Maßnahmen notwendig sind (Virginia Apgar selbst beschrieb die Herzfrequenz als aussagekräftigsten Parameter für die rasche Erholung eines deprimierten Neugeborenen) [106].

\section{Atmung}

Überprüfen Sie, ob das Neugeborene atmet. Wenn es atmet, beurteilen Sie die Atemfrequenz, die Atemtiefe und ob die Atemexkursionen seitengleich sind. Achten Sie auf Zeichen pathologischer Atemmuster, wie eine Schnappatmung oder Stöhnen (Knorksen).

\section{Herzfrequenz}

Die Herzfrequenz ist der beste klinische Parameter, um den Zustand eines Neugeborenen nach der Geburt zu beurteilen, und zeigt zudem am sensitivsten den Erfolg von unterstützenden Maßnahmen an. Die Herzfrequenz kann initial am schnellsten und zuverlässig durch die Auskultation über der Herzspitze mit dem Stethoskop [108] oder durch ein EKG-Monitoring beurteilt werden [109-112]. Das Tasten des Pulses an der Basis der Nabelschnur ist oft möglich, kann aber durchaus irreführend sein. Zuverlässig beurteilbar ist nur eine getastete Herzfrequenz $>$ 100/min [108]. Eine Beurteilung des klinischen Zustands allein kann zu einer Unterschätzung der Herzfrequenz führen $[108,109,113]$. Für reanimationspflichtige Neugeborene und/oder Neugeborene, die prolongiert beatmet werden müssen, liefern moderne Pulsoxymeter zuverlässige Herzfrequenzwerte [111]. Einige Studien zeigen jedoch, dass ein EKG, gerade in den ersten beiden Minuten nach der Geburt, die korrekte Herzfrequenz schneller und zuverlässiger als eine Pulsoxymetrie anzeigt [110-115]. Das EKG ersetzt jedoch nicht die Beurteilung der Sauerstoffsättigung des Neugeborenen durch eine Pulsoxymetrie.

\section{Hautkolorit}

Das Hautkolorit ist ein schlechter Parameter zur Beurteilung der Oxygenierung [116]. Diese sollte, wenn möglich, mittels Pulsoxymetrie erfasst werden. Ein gesundes Neugeborenes ist unmittelbar nach der Geburt zunächst zyanotisch und wird bei effektiver Spontanatmung innerhalb von $30 \mathrm{~s}$ zunehmend rosiger. Eine periphere Zyanose ist häufig und allein für sich kein Zeichen einer Hypoxie. Eine ausgeprägte, persistierende Blässe - trotz effektiver Ventilationen - kann ein Zeichen einer signifikanten Azidose, seltener auch einer Hypovolämie sein. Auch wenn die optische Beurteilung des Hautkolorits eine schlechte Methode zur Erfassung einer Zyanose ist, sollte sie nicht unterbewertet werden. Wenn Ihnen ein Neugeborenes zyanotisch erscheint, überprüfen Sie daher unbedingt die präduktale Oxygenierung des Neugeborenen durch Anlegen einer Pulsoxymetrie an der rechten Hand.

\section{Muskeltonus}

Ein deutlich hypotones Neugeborenes ist zumeist auch bewusstlos und benötigt respiratorische Unterstützung.

\section{Taktile Stimulation}

Das Abtrocknen des Neugeborenen ist gewöhnlich eine ausreichende Stimulation um eine effektive Spontanatmung anzuregen. Eine übertrieben kräftige Stimulation soll vermieden werden. Entwickelt das Baby unter kurzer taktiler Stimulation keine effektive Spontanatmung, sind weitere unterstützende Maßnahmen notwendig.

\section{Einteilung nach der initialen klinischen Beurteilung}

Anhand der initialen klinischen Beurteilung lassen sich Neugeborene in drei Gruppen einteilen:

\section{Gruppe 1 \\ - Suffiziente Atmung/Schreien \\ - Guter Muskeltonus \\ - Herzfrequenz > 100/min}

Diese Neugeborenen müssen nicht sofort abgenabelt werden. Sie benötigen außer Abtrocknen und Einwickeln in warme Tücher keine weiteren Maßnahmen. Das Neugeborene kann der Mutter übergeben werden. Durch den Hautkontakt mit ihr wird das Baby gewärmt, und beide werden zusätzlich mit einer Decke zugedeckt. Das Neugeborene kann zu diesem Zeitpunkt auch bereits erstmals an die Brust angelegt werden. Während dieser Phase 


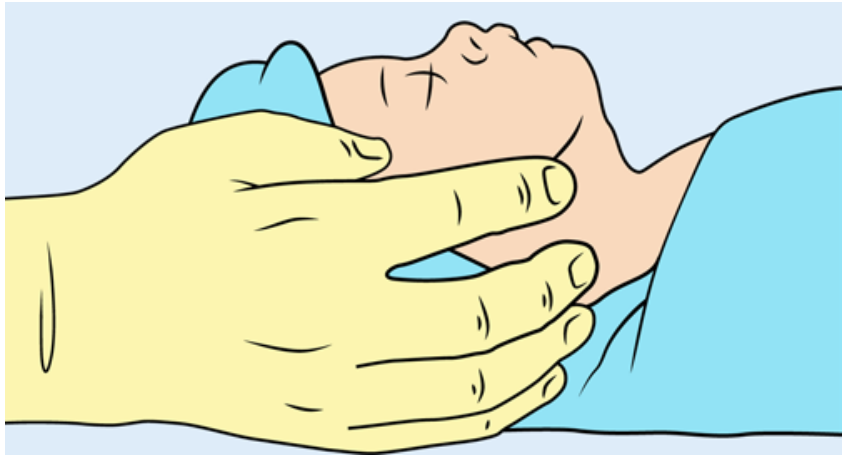

Abb. $2<$ Neutralposition des Kopfes beim Neugeborenen

muss allerdings unbedingt darauf geachtet werden, dass das Kind nicht auskühlt.

\section{Gruppe 2}

- Insuffiziente Spontanatmung oder Apnoe

- Normaler bis reduzierter Muskeltonus

- Herzfrequenz < 100/min

Diese Neugeborenen werden abgetrocknet und in warme Tücher gewickelt. Meist ist eine kurze Maskenbeatmung ausreichend. Einige Neugeborene benötigen jedoch auch eine längere Maskenbeatmung.

\section{Gruppe 3}

- Insuffiziente Spontanatmung oder Apnoe

- Schlaffer Muskeltonus (floppy)

- Bradykardie oder nicht nachweisbare Herzfrequenz

- Oft ausgeprägte Blässe als Zeichen einer schlechten Perfusion

Diese Neugeborenen werden abgetrocknet und in warme Tücher gewickelt. Sie müssen nach dem Öffnen der Atemwege unverzüglich beatmet werden. Möglicherweise benötigen diese Kinder im weiteren Verlauf auch Thoraxkompressionen, eventuell kann eine Medikamentengabe notwendig sein.

Eine sehr kleine Gruppe von Neugeborenen bleibt trotz adäquater Spontanatmung und guter Herzfrequenz hypoxämisch. Die Diagnosen sind hier vielfältig, infrage kommt z. B. ein kongenitales Vitium cordis, eine konnatale Pneumonie, ein Pneumothorax, eine Zwerchfellhernie oder ein Surfactantmangel.

Frühgeborene atmen zumeist spontan, zeigen aber häufig gleichzeitig Anzeichen einer Atemnot. Ist dies der Fall, sollen sie zunächst eine Atemunterstützung mittels CPAP („continuous positive airway pressure") erhalten.

\section{Die Reanimation des Neugeborenen - Newborn Life Support}

Mit Reanimationsmaßnahmen muss begonnen werden, wenn Sie bei der initialen Beurteilung feststellen, dass das Neugeborene keine suffiziente und regelmäßige Spontanatmung entwickelt hat oder die Herzfrequenz unter 100/min liegt (- Abb. 1).

Meist ist dann nach dem Öffnen der Atemwege lediglich eine kurze Maskenbeatmung notwendig, um die Lungen mit Luft zu füllen. Das Kind erholt sich darunter sofort. Bedenken Sie allerdings, dass alle weiteren Maßnahmen erfolglos bleiben werden, wenn diese ersten beiden Schritte, das Öffnen der Atemwege und die Belüftung der Lunge, nicht erfolgreich durchgeführt wurden.

\section{Atemwege}

Lagern Sie das Neugeborene in Rückenlage mit dem Kopf in Neutralposition (- Abb. 2). Zur optimalen Lagerung und Stabilisierung des Kopfes in Neutralposition kann die Platzierung eines $2 \mathrm{~cm}$ dicken Lakens oder Handtuchs unter den Schultern des Neugeborenen hilfreich sein.

Um die Atemwege eines hypotonen Neugeborenen zu öffnen, kann ein Esmarch-Handgriff oder die Verwendung eines oropharyngealen Tubus (GuedelTubus) in passender Größe sehr sinnvoll sein. Neugeborene, die beatmet werden, sollen in Rückenlage versorgt werden. Für die initiale Beurteilung und Routineversorgung im Kreißsaal können reife Neugeborene auch in Seitenlage positioniert werden [117].

Das routinemäßige oropharyngeale Absaugen des Neugeborenen ist nicht in allen Fällen notwendig [118]. Neugeborene müssen nur abgesaugt werden, wenn die Atemwege verlegt sind. Eine solche Verlegung kann aufgrund von Mekonium (selbst wenn das Neugeborene keine Mekoniumablagerungen auf der Haut zeigt), Blutkoageln, zähem Schleim oder Vernix bestehen. Wird ein Neugeborenes abgesaugt, ist zu bedenken, dass zu heftiges oropharyngeales Absaugen das Einsetzen einer suffizienten Spontanatmung verzögern und zu einem Laryngospasmus sowie zu einer vagusinduzierten Bradykardie führen kann [119-121].

\section{Mekonium}

Mehr als 30 Jahre lang bestand die Hoffnung, dass ein Absaugen von Mekonium aus den Atemwegen das Auftreten und den Schweregrad eines Mekoniumaspirationssyndroms (MAS) positiv beeinflussen könnte. Studien, die zu dieser Ansicht beitrugen, verglichen das Outcome von Neugeborenen, die abgesaugt wurden, mit historischen Kontrollgruppen [122, 123]. Andere Studien konnten keinerlei Vorteile dieses Vorgehens nachweisen [124, 125].

Leicht grünliches Fruchtwasser ist häufig und muss das versorgende Team im Allgemeinen nicht beunruhigen, da dies zumeist keine Auswirkungen auf die respiratorische Adaptationsphase nach der Geburt hat. Viel seltener findet sich zähes, grünes Fruchtwasser. Dies ist allerdings ein Hinweis für intrauterinen Stress des Neugeborenen und muss das Team immer in Alarmbereitschaft versetzen, da möglicherweise Reanimationsmaßnahmen notwendig werden. Zwei randomisierte Multizenterstudien haben gezeigt, dass die routinemäßige Intubation, verbunden mit einem trachealen Absaugen des vitalen Neugeborenen, die Inzidenz eines MAS nicht reduzieren konnte [126] und dass ein peripartales Absaugen nach Geburt des Kopfes und vor Geburt der Schultern ebenfalls zu keiner Verbesserung des Outcomes führt [127]. Daher wird bei mekoniumhaltigem Fruchtwas- 


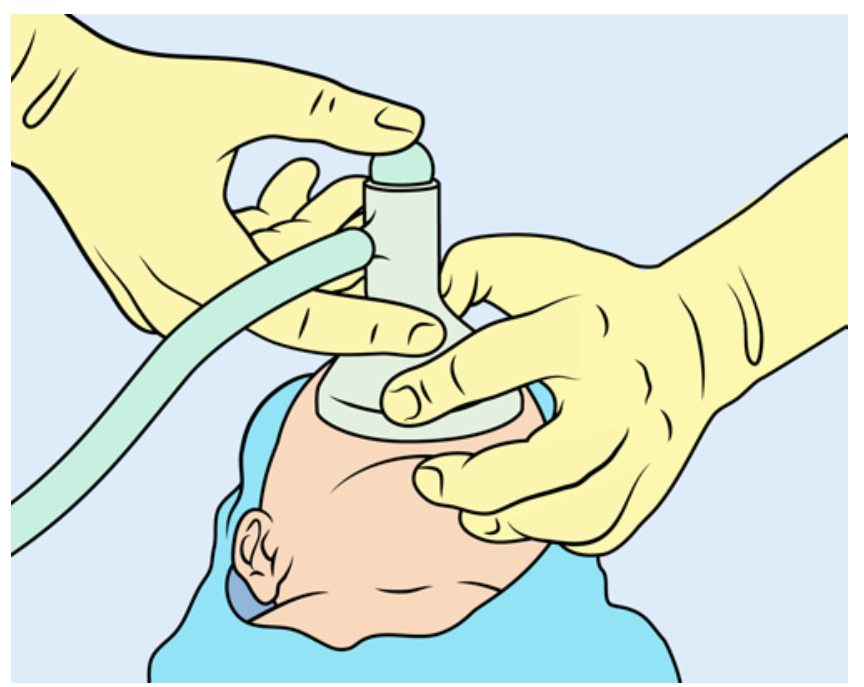

Abb. $3<$ Maskenventilation des Neugeborenen

ser weder das intrapartale Absaugen noch ein tracheales Einstellen und Absaugen eines vitalen Kindes empfohlen. Eine neue kleine RCT-Studie konnte beim nicht vitalen Neugeborenen keinen Unterschied in der Inzidenz eines MAS zwischen Kindern, die intubiert und tracheal abgesaugt wurden, und solchen, die nicht intubiert und abgesaugt wurden, nachweisen [128].

Handelt es sich um dickes, zähes Mekonium bei einem nicht vitalen Neugeborenen und wird eine Verlegung der Atemwege durch Mekonium vermutet, kann in diesem seltenen Fall eine Inspektion des Oropharynx und ein Absaugen unter Sicht in Erwägung gezogen werden. Eine routinemäßige tracheale Intubation wird bei mekoniumhaltigem Fruchtwasser und nicht vitalem Neugeborenen nicht mehr generell empfohlen und soll nur bei Verdacht auf eine wirkliche Obstruktion der Trachea mit Mekonium durchgeführt werden [128-132]. Entscheidend ist in diesen Situationen, bei einem nicht oder insuffizient atmenden Neugeborenen eine Beatmung nicht unnötig $\mathrm{zu}$ verzögern, sondern mit dieser bereits innerhalb der ersten Lebensminute zu beginnen. Für das Absaugen eignet sich ein 12-14-Ch-Absaugkatheter oder ein pädiatrischer Jankauer. Der Sog beim Absaugen sollte - $150 \mathrm{mmHg}$ nicht unterschreiten [133]. Eine routinemäßige Surfactantgabe oder eine Lavage mit Kochsalz oder Surfactant wird derzeit nicht empfohlen $[134,135]$.

\section{Initiale Beatmungshübe und assistierte Beatmung}

Nachdem das Neugeborene abgetrocknet, in Tücher gewickelt und die Atemwege geöffnet wurden, hat bei fehlender oder insuffizienter Spontanatmung die Belüftung der Lungen Priorität und darf nicht verzögert werden (• Abb. 3). Bei reifen Neugeborenen sollte die Beatmung mit Raumluft begonnen werden [136]. Das wichtigste Kriterium zur Beurteilung einer adäquaten Lungenentfaltung und -belüftung ist dabei der rasche Anstieg der Herzfrequenz. Kommt es zu keinem solchen Anstieg, muss überprüft werden, ob sich der Thorax adäquat hebt und senkt. Bei reifen Neugeborenen führen in der Regel spontane oder assistierte erste Atemzüge rasch zu einer funktionalen Residualkapazität (FRC) [137-141]. Welcher Beatmungsdruck, welche Inspirationszeit und welcher Flow zur Herstellung einer effektiven FRC optimal ist, ist bisher nicht festgelegt worden.

Für die ersten 5 Beatmungen soll der Inspirationsdruck über 2-3 s pro Beatmung konstant gehalten werden. Dies erleichtert im Allgemeinen die Entfaltung der Lunge [137, 142]. Der notwendige Druck zur Entfaltung der mit Flüssigkeit gefüllten Lunge liegt bei Neugeborenen, die Reanimationsmaßnahmen benötigen, zwischen 15 und $30 \mathrm{~cm} \mathrm{H}_{2} \mathrm{O}(1,5-2,9 \mathrm{kPa})$, durchschnittlich etwa bei $20 \mathrm{~cm} \mathrm{H}_{2} \mathrm{O}$ [137, 141, 142]. Für reife Neugeborene soll daher ein Spitzendruck von $30 \mathrm{~cm} \mathrm{H}_{2} \mathrm{O}$ und für Frühgeborene von $20-25 \mathrm{~cm} \mathrm{H}_{2} \mathrm{O}$ verwendet werden $[143,144]$.

Die Effektivität dieser ersten Beatmungen zeigt sich am besten an einem prompten Anstieg der Herzfrequenz oder an effektiven Thoraxhebungen. Kommt es nicht zum Anstieg der Herzfrequenz bzw. zu Thoraxhebungen, ist es möglicherweise notwendig, die Kopf- oder Maskenposition zu optimieren, nur selten ist ein höherer Beatmungsdruck notwendig.

Die meisten Neugeborenen, die nach der Geburt beatmet werden müssen, zeigen unter Ventilation der Lungen einen raschen Anstieg der Herzfrequenz, meist innerhalb von $30 \mathrm{~s}$. Steigt die Herzfrequenz an, aber das Neugeborene zeigt keine ausreichende Spontanatmung, wird mit 30 Beatmungen pro Minute weiterbeatmet, bis eine suffiziente Spontanatmung einsetzt. Die Inspirationszeit soll nun für die einzelnen Beatmungshübe bei einer Sekunde liegen.

Eine suffiziente Beatmung zeigt sich in der Regel an einem sofortigen Anstieg der Herzfrequenz bzw. daran, dass sich die Herzfrequenz unter der Beatmung stabil über 100/min hält. Reagiert das Neugeborene nicht entsprechend, liegt die Ursache in den meisten Fällen in einer ungenügenden Öffnung der Atemwege oder einer ineffektiven Beatmung. Achten Sie bei der Beatmung auf Thoraxbewegungen, die eine adäquate Entfaltung der Lungen anzeigen. Hebt sich der Thorax nicht, wurde die Lunge nicht geöffnet. Dies kann an einer Leckage der Beatmungsmaske, einer nicht korrekten Kopfposition oder an einer Obstruktion der Atemwege liegen [145-149]. Positionieren Sie in diesem Fall die Maske neu, um eine Leckage auszuschließen und/oder repositionieren Sie den Kopf des Kindes [145]. Alternativ kann mit Hilfe einer zweiten Person ein Zwei-Hände-Esmarch-Handgriff für die Maskenbeatmung bei reifen Neugeborenen und Frühgeborenen versucht werden, um die Leckage der Maske zu reduzieren [146, 147]. Ohne suffiziente Belüftung der Lungen werden Thoraxkompressionen nicht wirksam sein. Bevor mit diesen begonnen wird, muss daher unbedingt die effektive Ventilation der Lungen sichergestellt sein.

Eine Möglichkeit der Sicherung der AtemwegeistdieendotrachealeIntubation. 


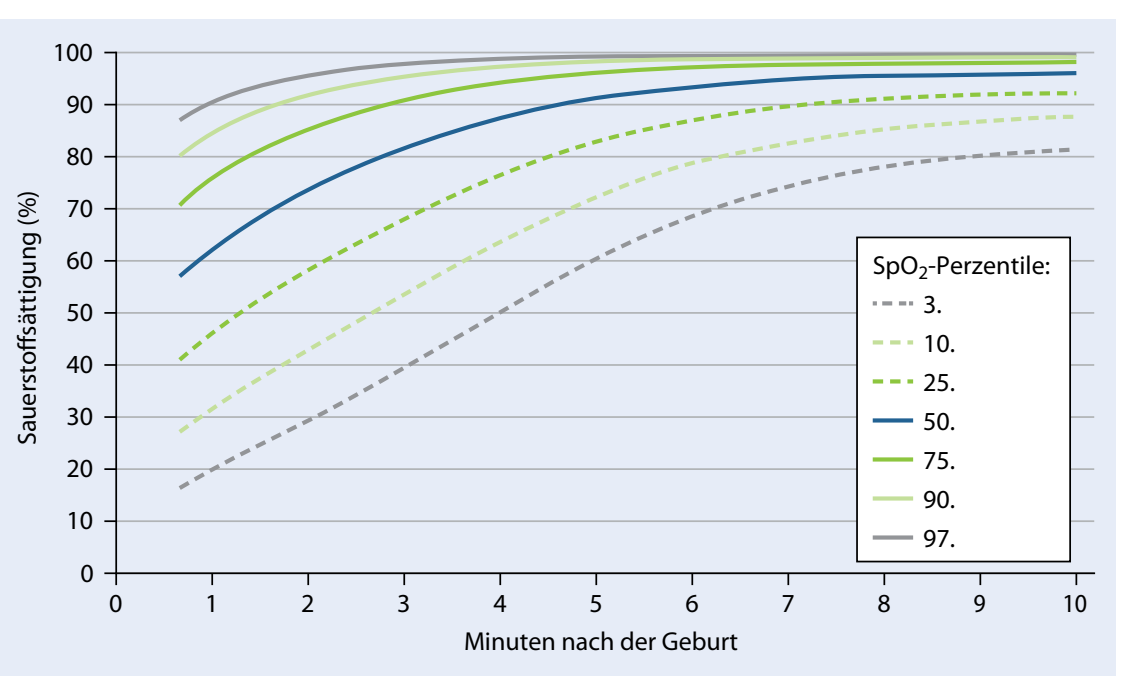

Abb. 4 ॥ Periphere Sauerstoffsättigung. Gesunde Neugeborene ohne medizinische Intervention. (Mit freundl. Genehmigung von Dawson et al. [157])

Diese benötigt jedoch Übung und Erfahrung. Ist niemand anwesend, der eine Intubation bei einem Neugeborenen durchführen kann, und kommt es zu keiner Stabilisierung der Herzfrequenz, muss erneut die Kopfposition überprüft und nochmals Beatmungen mit verlängerter Inspirationszeit durchgeführt werden. Währenddessen soll ein Helfer mit ausreichender Intubationserfahrung zur Hilfe gerufen werden. Fahren Sie mit der Beatmung fort, bis das Neugeborene eine suffiziente, regelmäßige Spontanatmung zeigt.

\section{Verlängerte Inspirationen $(\mathrm{SI}-$ „s sustained inflations") $>5 \mathrm{~s}$}

Einige tierexperimentelle Studien haben einen positiven Effekt auf die Bildung der funktionalen Residualkapazität gezeigt, wenn die Inspirationszeit während der initialen Beatmungen auf mehr als 2-3 s verlängert wurde $[150,151]$. Bei Durchsicht aller Studien bis 2015 finden sich drei RCT-Studien [152-154] und zwei Kohortenstudien, [144, 155], die zeigen konnten, dass bei deutlich verlängerten Inspirationszeiten seltener eine mechanische Beatmung notwendig war. Allerdings führte dies weder zu einer reduzierten Mortalität noch zur Verringerung einer bronchopulmonalen Dysplasie oder der Pneumothoraxwahrscheinlichkeit. Eine Kohortenstudie konnte zeigen, dass nach verlängerten Inspirationen seltener intubiert werden musste [144]. Bisher fehlen allerdings
Daten über die generelle Sicherheit, Details über die optimale Länge der verlängerten Inspirationen, den optimalen Beatmungsdruck und die Langzeiteffekte von verlängerten Inspirationen. Daher waren die COSTR-Mitglieder der Meinung, dass eine generelle Empfehlung für verlängerte Inspirationen $(>5 \mathrm{~s})$ in der Phase direkt nach der Geburt zum jetzigen Zeitpunkt nicht ausgesprochen werden kann $[1,2]$.

Verlängerte Inspirationen ( $>5 \mathrm{~s}$ ) sollten derzeit nur als Einzelfallentscheidung oder im Rahmen von Studien erwogen werden.

\section{Raumluft/Sauerstoff}

Reife Neugeborene. Eine Beatmung von reifen Neugeborenen soll immer mit einer Sauerstoffkonzentration von 21\% und nicht mit $100 \%$ begonnen werden. Kommt es trotz effektiver Beatmung zu keinem Anstieg der Herzfrequenz oder einer zufriedenstellenden Sauerstoffsättigung (idealerweise gemessen über eine Pulsoxymetrie), soll eine Erhöhung der Sauerstoffkonzentration in Erwägung gezogen werden, um eine adäquate präduktale Sättigung (Anmerkung der Übersetzer: gemessen an der rechten Hand) zu erreichen [156, 157]. Hohe Sauerstoffkonzentrationen sind mit einer erhöhten Mortalität und dem verzögerten Einsetzen der Spontanatmung verbunden [158]. Wird Sauerstoff in höherer Konzentration verwendet, soll diese daher so schnell wie möglich wieder reduziert werden [136, 159].

Frühgeborene. Für Frühgeborene vor der 35. Schwangerschaftswoche soll initial Raumluft oder eine niedrige Sauerstoffkonzentration (21-30\%) verwendet werden $[1,2,136,160]$. Sauerstoff soll so titriert werden, dass akzeptable präduktale Sauerstoffkonzentrationen erreicht werden, etwa der 25. Perzentile gesunder reifer Neugeborener direkt nach der Geburt entsprechend [156, 157] (• Abb. 4).

Eine Metaanalyse von 7 randomisierten Studien, die den Start von Reanimationsmaßnahmen mit einer hohen Sauerstoffkonzentration $(>65 \%)$ und einer geringen Sauerstoffkonzentration (21-30\%) verglichen hat, konnte keine Verbesserung in Bezug auf Überlebensrate [159, 161-166], bronchopulmonale Dysplasie $[159,162,164-166]$, intraventrikuläre Blutungen (IVH) $[159,162,165,166]$ oder Frühgeborenenretinopathie (ROP) [159, $162,166]$ bei der Verwendung von höher konzentriertem Sauerstoff zeigen. Allerdings zeigten sich erhöhte Marker eines oxidativen Stresses [159].

Pulsoxymetrie. Eine moderne Pulsoxymetrie, mit neonatologischen Sensoren, erlaubt eine zuverlässige Anzeige der Herzfrequenz und der peripheren Sättigung innerhalb von 1-2 min nach der Geburt (• Abb. 4) [167, 168]. Zuverlässige präduktale Sättigungswerte lassen sich bei über $90 \%$ der gesunden Neugeborenen, etwa $80 \%$ der Frühgeborenen und 80-90 \% der Neugeborenen, die Reanimationsmaßnahmen benötigten, innerhalb von 2 min nach der Geburt ableiten [167]. Unbeeinträchtigte, reife Neugeborene haben unter der Geburt eine arterielle Sauerstoffsättigung $\left(\mathrm{S}_{\mathrm{p}} \mathrm{O}_{2}\right)$ von etwa $60 \%$ (gemessen auf Normalhöhennull) [169]. Diese steigt innerhalb von $10 \mathrm{~min}$ auf Werte über $90 \%$ an (• Abb. 4; [156]). Die 25. Perzentile der postnatalen $\mathrm{S}_{\mathrm{p}} \mathrm{O}_{2^{-}}$ Werte liegt bei etwa $40 \%$ bei Geburt und steigt in der zehnten Lebensminute auf etwa $80 \%$ [157]. Neugeborene, die durch Kaiserschnitt entbunden [170], in größerer Höhe geboren [171] oder spät abgenabelt werden, zeigen noch niedrigere 
Werte [172]. Frühgeborene benötigen teilweise mehr Zeit, um $\mathrm{S}_{\mathrm{p}} \mathrm{O}_{2}$-Werte über $90 \%$ zu erreichen [157].

Die Pulsoxymetrie soll in der Neugeborenenversorgung eingesetzt werden, um exzessive Sauerstoffgaben zu vermeiden und die Sauerstofftherapie sinnvoll steuern zu können. Periphere Sättigungen über den angestrebten Werten sollen daher prompt zur Reduktion der Sauerstoffkonzentration führen.

\section{Positiver endexpiratorischer Druck (PEEP)}

Neugeborene und Frühgeborene, die trotz initialer Beatmungen zum Öffnen der Lunge keine Spontanatmung entwickeln, müssen weiter beatmet werden. Frühgeborene sollen dann mit einem positiven endexpiratorischen Druck (PEEP) von etwa $5 \mathrm{~cm} \mathrm{H}_{2} \mathrm{O}$ beatmet werden [173].

Tierexperimentelle Studien zeigen, dass hohe Tidalvolumen schon bei den ersten Beatmungen nach der Geburt zu einer Schädigung der frühgeborenen Lunge führen können [174]. Die Applikation eines PEEP direkt nach der Geburt scheint dagegen vor Lungenschädigung zu schützen $[175,176]$. Andere Studien bestätigten diesen positiven Effekt hingegen nicht [177]. Eine PEEP-Applikation erleichtert allerdings das Öffnen der Lunge, verbessert die Lungencompliance und den Gasaustausch [178-180]. Zwei RCT-Studien mit Neugeborenen konnten keine Verbesserung der Mortalität, keine geringere Wahrscheinlichkeit einer Reanimation und keine seltenere Inzidenz einer bronchopulmonalen Dysplasie zeigen, wobei diese Studien nicht genug statistische Aussagekraft bezüglich dieser Outcome-Parameter hatten $[181,182]$. Allerdings schien bei einer dieser Studien der zusätzlich notwendige Sauerstoffbedarf unter PEEP-Gabe geringer zu sein [182].

\section{Hilfsmittel zur assistierten Beatmung}

Effektive Beatmungen lassen sich mit einem Beutel, dessen Füllung abhängig vom Gasfluss ist (Anästhesiebeutel - Anmerkung der Übersetzer), mit einem sich selbst füllenden Beatmungsbeutel oder mit einem T-Stück-System, das eine Regulierung des applizierten Spitzendrucks erlaubt, erreichen [181-185]. Selbstfüllende Beatmungsbeutel besitzen Überdruckventile, die sich, abhängig vom Gasfluss, öffnen. Unter brüsker Beatmung kann der applizierte Beatmungsdruck allerdings durchaus den vom Hersteller angegebenen Spitzendruck übersteigen [186, 187]. Die Verwendung eines Systems mit T-Stückerlaubtim Gegensatzzuherkömmlichen Beatmungsbeuteln eine bessere Kontrolle des applizierten Spitzendrucks und des Tidalvolumens, außerdem erleichtert es die Beatmung mit verlängerten Inspirationszeiten [187-190]. Allerdings ist die klinische Bedeutung dieser Beobachtungen noch nicht eindeutig klar. Es erfordert mehr Übung, einen ausreichenden Beatmungsdruck mithilfe eines anästhesiologischen Beutels (dessen Füllung abhängig vom Gasfluss ist) zu erreichen als mit einem selbstfüllenden Beatmungsbeutel [191]. Sowohl Anästhesiebeutel als auch selbstfüllende Beatmungsbeutel sowie mechanische T-Stück-Modelle sind so konstruiert, dass sich ein bestimmter Beatmungsdruck applizieren und begrenzen lässt. Sie sind daher prinzipiell alle zur Beatmung von Neugeborenen geeignet. Allerdings können nur selbstfüllende Beatmungsbeutel auch ohne Gasfluss verwendet werden. Diese sind jedoch nicht für eine Atemunterstützung mittels CPAP und kaum für eine Beatmung mit PEEP geeignet, selbst wenn sie ein sogenanntes „PEEP-Ventil“ besitzen [189, 192-195].

Mithilfe von Atemfunktionsmonitoren lassen sich Beatmungsdruck und Tidalvolumen messen und anzeigen [189, 192195], mit einer $\mathrm{CO}_{2}$-Messung kann man ausgeatmetes $\mathrm{CO}_{2}$ bestimmen [197, 198]. Diese Monitore wurden bei der Versorgung von Neugeborenen bereits verwendet, um Beatmungen zu beurteilen; allerdings gibt es bisher keine Daten darüber, ob deren Verwendung zu einer OutcomeVerbesserung führt. Bisher ist ebenso unklar, ob die Verwendung solcher Monitore einen Vorteil gegenüber der alleinigen klinischen Beurteilung von Beatmungen bedeutet oder ob die Verwendung möglicherweise mit Risiken verbunden ist. Studien, die eine Messung des exspiratorischen $\mathrm{CO}_{2}$ im Zusammenhang mit an- deren Hilfsmitteln zur Beatmung (z. B. Wendl Tuben, Larynxmasken) untersucht haben, gibt es bisher ebenfalls nicht.

\section{Beatmungsmaske im Vergleich zu nasalen Prongs}

Bei der Verwendung einer Beatmungsmaske bei Neugeborenen kann das Abdichten mitunter zu Problemen führen [145-148]. Um dies zu vermeiden, werden in einigen Institutionen nasopharyngeale Prongs bzw. ein nasopharyngealer Tubus für die Beatmung verwendet. Zwei randomisierte Studien bei Frühgeborenen konnten allerdings keinen Unterscheid beider Methoden bezüglich der Effektivität der Beatmung nachweisen [199, 200].

\section{Larynxmasken (LMA)}

Eine Larynxmaske kann für die Beatmung eines Neugeborenen verwendet werden, vor allem wenn eine Maskenbeatmung oder auch eine Intubation nicht gelingt oder nicht möglich ist. Sie kann bei Neugeborenen $>2000$ g bzw. $\geq 34$ Schwangerschaftswochen als Alternative zu einer Maskenbeatmung in Erwägung gezogen werden [201]. In einer kürzlich publizierten ungeblindeten RCT-Studie führte das Training mit einem Modell einer Larynxmaske dazu, dass weniger Intubationen nötig waren und eine geringere Zahl von Neugeborenen auf die Intensivstation aufgenommen werden mussten im Vergleich zu Neugeborenen, die maskenbeatmet wurden [201]. Allerdings gibt es bisher nur wenig Evidenz für eine Verwendung in der Gruppe der Neugeborenen $<2000 \mathrm{~g}$ bzw. $<34$ Schwangerschaftswochen. Eine Larynxmaske kann auch als Alternative für eine Intubation bei Neugeborenen $>2000$ g bzw. $\geq 34$ Schwangerschaftswochen in Erwägung gezogen werden [201-206]. Sie wird für die Beatmung von Früh- und Neugeborenen $\geq 34$ Schwangerschaftswochen empfohlen, wenn eine Intubation nicht gelingt oder nicht möglich ist. Allerdings wurde die Verwendung einer Larynxmaske noch nicht im Rahmen von Geburten mit mekoniumhaltigem Fruchtwasser, während der Durchführung von Thoraxkompressionen oder für die notfallmäßige intratracheale Gabe von Medikamenten untersucht. 


\begin{tabular}{|c|c|}
\hline Gestationswoche & $\begin{array}{l}\text { Tubustiefe ab } \\
\text { Unterlippe (in cm) }\end{array}$ \\
\hline $23-24$ & 5,5 \\
\hline $25-26$ & 6,0 \\
\hline $27-29$ & 6,5 \\
\hline $30-32$ & 7,0 \\
\hline $33-34$ & 7,5 \\
\hline $35-37$ & 8,0 \\
\hline $38-40$ & 8,5 \\
\hline $41-43$ & 9,0 \\
\hline
\end{tabular}

\section{Endotracheale Intubation}

Eine endotracheale Intubation (• Tab. 1) kann zu unterschiedlichen Zeitpunkten während einer Neugeborenenreanimation in Erwägung gezogen werden:

- wenn ein Absaugen der unteren Atemwege bei Verdacht auf eine eine tracheale Verlegung notwendig ist,

- wenn sich das Neugeborene trotz Repositionierung des Kopfes und/oder der Maske weiter nicht maskenbeatmen lässt oder eine längere Beatmungsdauer abzusehen ist,

- wenn Thoraxkompressionen durchgeführt werden,

- in speziellen Situationen (wie z. B. bei einer kongenitalen Zwerchfellhernie oder für eine tracheale Surfactantgabe).

Die Intubation und der ideale Zeitpunkt dazu werden von den Fähigkeiten und der Erfahrung des versorgenden Teams abhängig sein. Die entsprechende Tubustiefe in Abhängigkeit von der Gestationswoche (Anmerkung der Übersetzer: bei oraler Intubation) findet sich in $\bullet$ Tab. 1 [207]. An dieser Stelle soll darauf hingewiesen werden, dass die Stimmbandmarkierungen, die die korrekte Intubationstiefe erleichtern sollen, von Hersteller zu Hersteller beträchtlich variieren [208].

Die endotracheale Tubuslage und die Intubationstiefe müssen bei der Intubation unter direkter Sicht überprüft werden. Ein prompter Anstieg der Herzfrequenz nach der Intubation unter Beatmung ist ein gutes Zeichen für die endotracheale Tubuslage [209]. Der Nachweis von $\mathrm{CO}_{2}$ in der Ausatemluft ist ebenfalls eine effektive Methode zum Nachweis der endotrachealen Tubuslage bei Neugeborenen, selbst bei sehr kleinen Frühgeborenen (VLBW, ,very low birth weight infants") [210-213]. Mehrere Neonatalstudien zeigen, dass der Nachweis von ausgeatmetem $\mathrm{CO}_{2}$ unter Spontankreislauf schneller und zuverlässiger eine tracheale Tubuslage anzeigt, als dies durch eine klinische Beurteilung allein möglich ist [212-214]. Lässt sich kein $\mathrm{CO}_{2}$ nachweisen, macht dies eine ösophageale Tubuslage sehr wahrscheinlich [210, 212]. Allerdings wurden im Herz-Kreislauf-Stillstand [210] und bei sehr kleinen Frühgeborenen (VLBW) falsch-negative Ergebnisse trotz beschriebener Effektivität der Methode berichtet [215]. Neugeborene, die intensive Reanimationsmaßnahmen benötigten, wurden aus den Studien ausgeschlossen. Falsch-positive Ergebnisse sind bei kolorimetrischen $\mathrm{CO}_{2}$-Detektoren möglich, wenn diese mit Adrenalin, Surfactant oder Atropin in Kontakt kommen [198].

Eine zu geringe oder fehlende pulmonale Perfusion oder eine Obstruktion der Trachea kann, trotz korrekter endotrachealer Tubuslage, einen Nachweis von ausgeatmetem $\mathrm{CO}_{2}$ verhindern. Bei fast allen Patienten, die sich nicht im Kreislaufstillstand befinden, kann eine endotracheale Tubuslage durch den Nachweis von exspiratorischem $\mathrm{CO}_{2}$ korrekt nachgewiesen werden [211]. Bei einem kritisch kranken Neugeborenen mit reduziertem Herzminutenvolumen kann der fehlende $\mathrm{CO}_{2}$-Nachweis unter Umständen allerdings zu einer unnötigen Extubation führen. Weitere klinische Zeichen einer korrekten Tubuslage sind das Beschlagen des Tubusinneren während der Exspiration und das Heben und Senken des Brustkorbs während der Beatmung, allerdings wurde dies bisher nicht systematisch bei Neugeborenen untersucht.

Der Nachweis von $\mathrm{CO}_{2}$ in der Ausatemluft in Ergänzung zur klinischen Beurteilung wird als zuverlässigste Methode empfohlen, um bei Neugeborenen mit Spontankreislauf die tracheale Tubuslage nachzuweisen $[3,4]$.

\section{Kontinuierlicher positiver Atemwegsdruck (CPAP)}

Spontan atmende Frühgeborene, die Atemnot zeigen, sollten eher eine Atemunterstützung mittels CPAP erhalten, als intubiert zu werden. Drei RCT-Studien mit insgesamt 2358 Frühgeborenen $<30$ Schwangerschaftswochen haben gezeigt, dass CPAP gegenüber initialer Intubation mit folgender Beatmung vorteilhaft ist, um Intubationshäufigkeit und Beatmungsdauer zu reduzieren. Im KurzzeitOutcome zeigten sich zudem keine Nachteile [216-218]. Bisher gibt es nur wenige Daten für die Verwendung von CPAP bei reifen Neugeborenen nach der Geburt, und es werden weitere Studien notwen$\operatorname{dig}[219,220]$.

\section{Kreislaufunterstützung}

Eine Unterstützung des Kreislaufs durch Thoraxkompressionen kann nur wirksam sein, wenn die Lunge zuvor erfolgreich belüftet wurde. Beginnen Sie mit Thoraxkompressionen, wenn die Herzfrequenz trotz effektiver Beatmung unter 60/ min liegt. Effektive Beatmungen sind die wichtigste und häufig einzig notwendige Maßnahme für die erfolgreiche Reanimation eines Neugeborenen. Da eine Beatmung allerdings durch Thoraxkompressionen behindert werden kann, ist es entscheidend, zunächst sicherzustellen, dass sie effektiv ist, bevor Thoraxkompressionen durchgeführt werden.

Die effektivste Technik für Thoraxkompressionen beim Neugeborenen ist die 2-Daumen-Technik. Platzieren Sie hierfür zwei Daumen nebeneinander über dem unteren Drittel des Brustbeins. Umgreifen Sie mit den Fingern den gesamten Brustkorb und unterstützen Sie so den Rücken des Kindes (• Abb. 5; [221-224]). Mit der 2-Daumen-Technik können ein höherer systemischer Blutdruck und ein höherer koronarer Perfusionsdruck als mit der in den Leitlinien 2010 ebenfalls noch empfohlenen 2-Finger-Technik erreicht werden. Außerdem ist diese Technik weniger ermüdend [222-234]. In einer Simulationsstudie mit Reanimationspuppen führte das Platzieren der Daumen über- 


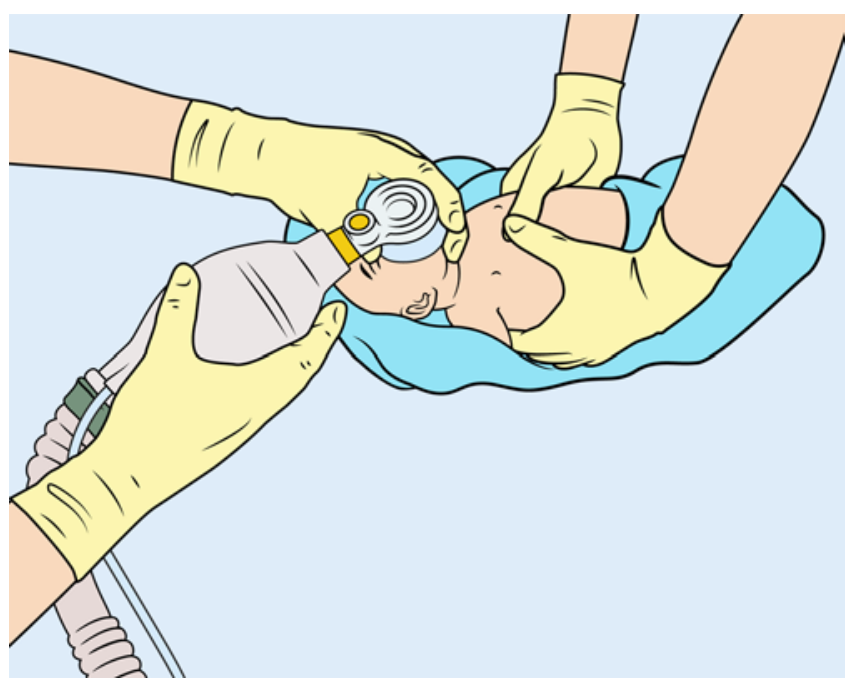

Abb. $5<$ Beatmungen und Thoraxkompressionen beim Neugeborenen

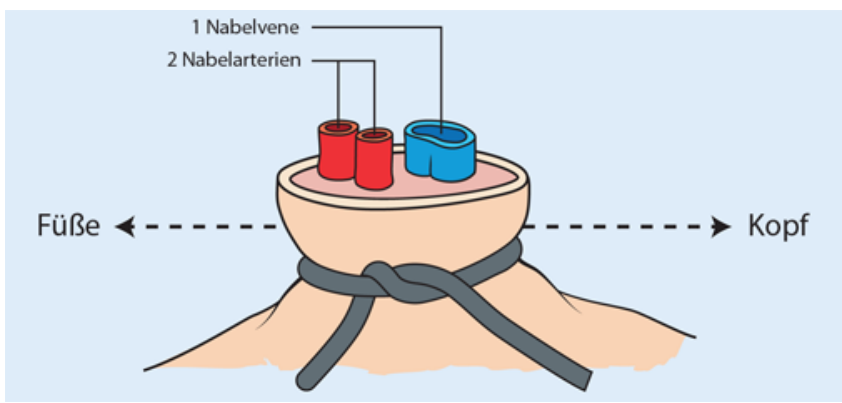

in der Ausatemluft und die der peripheren Sättigung mittels Pulsoxymetrie haben sich in Studien als sinnvoll erwiesen, um eine Wiederherstellung eines Spontankreislaufs festzustellen [256-260], allerdings gibt es derzeit keine ausreichende Evidenz, um eine bestimmte MonitoringMethode für den klinischen Gebrauch zu empfehlen [1,2].

\section{Medikamente}

Die Gabe von Medikamenten ist bei der Reanimation eines Neugeborenen nur sehr selten erforderlich. Eine Bradykardie wird beim Neugeborenen in den meisten Fällen entweder durch eine inadäquate Ventilation oder eine schwere Hypoxie verursacht. Die wichtigste Maßnahme zur Behebung der Bradykardie ist daher eine effektive Beatmung. Bleibt die Herzfrequenz trotz suffizienter Beatmungen und schließlich Thoraxkompressionen und Beatmungen im Wechsel unter 60/min, soll die Gabe von Medikamenten erwogen werden. Diese sollen idealerweise über einen Nabelvenenkatheter gegeben werden (• Abb. 6).

mit zwei Arterien un einer Vene einander zu effektiveren Thoraxkompressionen als das Platzieren der Daumen nebeneinander, allerdings führte es zu schnellerer Ermüdung [235]. Das Brustbein soll um ein Drittel des anterioposterioren Thoraxdurchmessers komprimiert werden und muss nach jeder Kompression wieder in seine Ausgangsposition zurückehren [225, 236-240].

Führen Sie Thoraxkompressionen und Beatmungen in einem Verhältnis von 3:1 durch. Bei einer Kompressionsfrequenz von etwa 120/min können etwa 90 Kompressionen und 30 Beatmungen pro Minute erreicht werden [241-246]. Eine gegenüber der Kompressionsphase gering verlängerte Relaxationsphase bietet theoretische Vorteile [247]. Letztlich ist die Qualität der Druckmassagen und der Beatmungen allerdings wahrscheinlich entscheidender als deren Frequenz. In jedem Fall sollen Thoraxkompressionen und Beatmungen koordiniert durchgeführt werden, um zu vermeiden, dass sie zusammentreffen [248]. Da in den meisten Fällen ein behinderter pulmonaler Gasaustausch die eigentliche Ursache für eine Kreislaufinsuffizienz beim Neugeborenen ist, wird für die Reanimation direkt nach der Geburt weiter ein KompressionsVentilations-Verhältnis von 3:1 empfohlen. Besteht der Verdacht auf eine kardiale Ursache für die Reanimationspflichtigkeit, kann das versorgende Team zugunsten der Thoraxkompressionen auch z. B. ein Verhältnis von 15 Thoraxkompressionen zu 2 Beatmungen erwägen.

Werden für die Reanimation eines Neugeborenen Thoraxkompressionen notwendig, wurde bis dahin wahrscheinlich mit einer niedrigen Sauerstoffkonzentration beatmet. Es erscheint dann im Verlauf durchaus plausibel zu sein, die Sauerstoffkonzentration auf $100 \%$ $\mathrm{zu}$ erhöhen. Allerdings gibt es keine Studien am Menschen, die dieses Vorgehen unterstützen. Tierexperimentelle Studien hingegen zeigen keinen Vorteil von Beatmungen mit $100 \%$ Sauerstoff während einer Reanimation [249-255].

Überprüfen Sie nach 30 s Thoraxkompressionen und Ventilationen und im weiteren Verlauf regelmäßig die Herzfrequenz. Beenden Sie die Thoraxkompressionen erst, wenn die Herzfrequenz über 60/min liegt. Die Messung von $\mathrm{CO}_{2}$

\section{Adrenalin}

Auch wenn die Datenlage aus Studien am Menschen nicht eindeutig ist, scheint die Verwendung von Adrenalin sinnvoll, wenn die Herzfrequenz trotz adäquater Beatmung und suffizienter Thoraxkompressionen nicht über $60 / \mathrm{min}$ ansteigt. Die empfohlene intravenöse Dosis beträgt $10 \mu \mathrm{g} / \mathrm{kg} \mathrm{KG}(0,1 \mathrm{ml} / \mathrm{kg} \mathrm{KG}$ der 1:10.000-Lösung) und soll so schnell wie möglich intravenös verabreicht werden. $[1,2,4]$ Sind weitere Gaben notwendig, sollen in der Folge 10-30 $\mu \mathrm{g} / \mathrm{kg}$ KG (0,10,3 ml/kg KG der 1:10.000-Lösung) pro Dosis verabreicht werden.

Eine endotracheale Verabreichung wird nicht empfohlen. Wird Adrenalin dennoch endotracheal gegeben, sind sehr wahrscheinlich Dosen von 50-100 $\mu \mathrm{g} /$ $\mathrm{kg} \mathrm{KG}$ notwendig [3, 7, 136, 261-265]. Allerdings wurde bisher weder die Sicherheit noch die Effektivität dieser höheren endotrachealen Dosis untersucht. Die hohe Dosis darf in keinem Fall intravenös gegeben werden. 


\section{Natriumbikarbonat}

Kommt es unter suffizienter Beatmung und effektiven Thoraxkompressionen nicht wieder zu einem Spontankreislauf, lässt sich die Myokardfunktion möglicherweise durch die Korrektur einer bestehenden myokardialen Azidose verbessern und so ein Spontankreislauf herstellen. Um eine Routinegabe von Natriumbikarbonat während der Reanimation eines Neugeborenen zu empfehlen, fehlen die entsprechenden Daten. Natriumbikarbonat ist hyperosmolar und führt zu einer Kohlendioxidfreisetzung. Dies kann die myokardiale und zerebrale Funktion beeinträchtigen. Während einer kurzen Reanimation wird die Gabe von Natriumbikarbonat daher nicht empfohlen. Im Rahmen eines prolongierten Kreislaufstillstands, der auf andere Maßnahmen nicht reagiert, soll die Gabe von Natriumbikarbonat nur unter effektiven Ventilationen und Thoraxkompressionen erwogen werden. Natriumbikarbonat kann unter diesen Umständen langsam intravenös in einer Dosis von 1-2 mmol/kg KG gegeben werden.

\section{Flüssigkeitsgabe}

Bei Verdacht auf einen neonatalen Blutverlust oder Zeichen eines Schocks (Blässe, schlechte periphere Durchblutung, schwache Pulse) soll eine Flüssigkeitsgabe erwogen werden, wenn das Neugeborene auf adäquate Reanimationsmaßnahmen nicht anspricht [266]. Dies ist allerdings sehr selten der Fall. Ist bei einem Blutverlust kein geeignetes Blut verfügbar (d. h. bestrahltes, leukozytendepletiertes, 0 -Rhnegatives Erythrozytenkonzentrat), sind zur Erhöhung des intravasalen Volumens isotonisch-kristalloide Lösungen albuminhaltigen vorzuziehen, um das intravasale Volumen zu erhöhen. Initial soll ein Flüssigkeitsbolus von $10 \mathrm{ml} / \mathrm{kg}$ KG verabreicht werden. Zeigt diese Maßnahme Erfolg, können im Verlauf eventuell wiederholte Bolusgaben notwendig sein, um die Verbesserung zu erhalten. Bei der Stabilisierung oder Reanimation von Frühgeborenen sind selten Flüssigkeitsbolusgaben notwendig. Eine schnelle Gabe von großen Volumenmengen ist hier mit intraventrikulären und pulmonalen Blutungen assoziiert.

\section{Beendigung von bzw. Verzicht} auf Reanimationsmaßnahmen

Mortalität und Morbidität von Neugeborenen variieren je nach Region und Verfügbarkeit von medizinischen Ressourcen [267]. Sozialwissenschaftliche Studien zeigen, dass Eltern sich generell eine bessere Einbindung in Entscheidungsprozesse bezüglich des Beginns oder auch des Fortführens bzw. der Beendigung von lebenserhaltenden Maßnahmen bei schwer beeinträchtigten Neugeborenen wünschen [268]. Dabei gibt es unterschiedliche Auffassungen bei medizinischem Personal, Eltern und in der Gesellschaft über Vorund Nachteile der medizinischen Therapie und wie weit Intensivmedizin generell bei schwerstkranken Neugeborenen gehen sollte [269, 270]. Lokale Überlebensund Outcome-Daten stellen daher wichtige Parameter für eine seriöse Beratung von Eltern dar. Von Bedeutung scheinen klare institutionelle Richtlinien über das Vorgehen an der Grenze der Lebensfähigkeit zu sein. Eine aktuelle Studie konnte bei Bestehen klarer Richtlinien eine Outcome-Verbesserung der überlebenden Kinder zeigen [271].

\section{Beendigung von Reanimationsmaßnahmen}

Regionale und nationale Gremien legen Empfehlungen zur Beendigung von Reanimationsmaßnahmen fest. Ist bei einem gerade geborenen Kind keine Herzfrequenz nachweisbar und ist sie auch nach 10 min Reanimationsmaßnahmen nicht nachweisbar, kann es angemessen sein, eine Beendigung der Wiederbelebungsmaßnahmen zu erwägen. Die Entscheidung, ob eine Reanimation nach $10 \mathrm{~min}$ ohne erkennbare Herzfrequenz fortgeführt wird, ist oft komplex und wird durch die weiteren Umstände bestimmt. Es fließen eine Vielzahl von Variablen ein, wie die Ätiologie des Kreislaufstillstands, das Gestationsalter, die Aussicht auf Reversibilität des Zustands, die Verfügbarkeit therapeutischer Hypothermie und zuvor von den Eltern geäußerte Ansichten über ein vertretbares Risiko einer Morbidität des Kindes [267, 272-276]. Die Entscheidung soll daher individuell getroffen werden. Liegt die Herzfrequenz nach der Geburt unter 60/min und kommt es trotz ad- äquater Reanimationsmaßnahmen nach 10 bis 15 min nicht zu einem signifikanten Anstieg der Herzfrequenz, ist die Entscheidung zur Fortführung oder Beendigung dieser Maßnahmen deutlich schwieriger. Für diese Situation gibt es keine ausreichenden Daten über die Prognose eines Kindes, um klare Empfehlungen für ein Weiterführen oder einen Abbruch der Reanimationsmaßnahmen zu geben.

\section{Verzicht auf Reanimationsmaßnahmen}

Es gibt Umstände, bei welchen die Prognose eines Neugeborenen mit einer hohen Mortalität und einem schlechten Outcome verbunden ist. Hier kann es vertretbar sein, auf Reanimationsmaßnahmen $\mathrm{zu}$ verzichten, insbesondere dann, wenn die Gelegenheit zur vorherigen Beratung mit den Eltern besteht [38, 272, 277-282]. Leider gibt es derzeit keine Evidenz für die Verwendung der verfügbaren prognostischen Scores auf alleiniger Grundlage des Gestationsalters für Frühgeborene $<25$ Gestationswochen.

Jeder Fall muss daher vom geburtshilflichen und neonatologischen Team individuell betrachtet werden und es ist wichtig Entscheidungen in schlüssiger und koordinierter Vorgehensweise gemeinsam mit den Eltern zu treffen [283]. Eine Reanimation nicht zu beginnen oder lebenserhaltende Maßnahmen während oder nach einer Reanimation zu beenden, wird im Allgemeinen als ethisch gleichwertige Entscheidung angesehen. Daher sollte nicht gezögert werden, lebenserhaltende Maßnahmen auch im weiteren Verlauf noch zu beenden, wenn ein Überleben ohne schwerste funktionelle Störungen unwahrscheinlich ist. Die folgenden Leitlinien müssen vor dem Hintergrund regional unterschiedlicher Daten zum Outcome interpretiert werden:

- Reanimationsmaßnahmen sollten nicht begonnen werden, wenn Gestationsalter, Geburtsgewicht und/oder kongenitale Fehlbildungen mit an Sicherheit grenzender Wahrscheinlichkeit mit einem frühen Tod verbunden sind und die sehr wenigen Überlebenden eine inakzeptabel hohe Morbidität aufweisen [38, 277, 284]. Beispiele aus der publizierten Literatur wären z. B. Frühgeborene 
mit einem Gestationsalter unter 23

Schwangerschaftswochen und/oder einem Geburtsgewicht unter $400 \mathrm{~g}$ sowie Anomalien wie eine Anenzephalie oder eine nachgewiesene Trisomie 13 oder 18 .

- Der Beginn von Reanimationsmaßnahmen ist fast immer angezeigt, wenn eine hohe Überlebensrate und eine vertretbare Morbidität wahrscheinlich sind. Darunter fallen grundsätzlich Frühgeborene ab der 25. Gestationswoche (es sei denn, es bestehen Hinweise auf schwerwiegende fetale Probleme, wie eine schwere intrauterine Infektion oder eine Hypoxie bzw. Ischämie) sowie die Mehrzahl der kongenitalen Fehlbildungen.

- Ist die Prognose für ein Kind aufgrund einer grenzwertigen Überlebensrate mit relativ hoher Morbidität ungewiss und sind die für das Kind anzunehmenden Belastungen hoch, sollten die Wünsche der Eltern bezüglich einer Reanimation berücksichtigt und unterstützt werden [283].

- Wird entschieden, eine Reanimation nicht zu beginnen oder zu beenden, steht für das Kind und die Familie nun ein Sterben ohne Schmerzen und in Würde im Vordergrund der Betreuung.

\section{Kommunikation mit den Eltern}

Das versorgende Team soll die Eltern unbedingt über den Zustand des Neugeborenen im Verlauf unterrichten. Halten Sie sich bei der Geburt an lokale Absprachen, und übergeben Sie das Neugeborene so frühzeitig wie möglich der Mutter. Sind Reanimationsmaßnahmen notwendig, sollen die Eltern über die durchgeführte Behandlung und die Gründe dafür aufgeklärt werden.

Die europäischen Leitlinien unterstützen die Anwesenheit der Eltern während einer Reanimation [285]. In den letzten Jahren machen medizinische Teams mehr und mehr von dieser Empfehlung Gebrauch, und Familienangehörige bekommen zunehmend die Möglichkeit, bei der Reanimation ihres Kindes dabeizubleiben. Dies gilt im Besonderen auch für die Reanimation im Kreißsaal bzw.
Sectio-OP. Dem Wunsch der Eltern, bei Reanimationsmaßnahmen dabei zu sein, soll daher, wann immer dies möglich ist, nachgekommen werden [286].

Dabei sollen die Mitglieder des versorgenden Teams gemeinsam mit der Familie ohne Zwang oder Druck entscheiden, welche Personen während der Reanimation dabei sein können. Es wird empfohlen, dass ein Teammitglied während der Reanimationsmaßnahmen ausschließlich für die Betreuung der Familienmitglieder zuständig ist. Ist das nicht möglich, bedeutet dies allerdings nicht zwangsläufig, dass sie nicht anwesend sein können. Mit den engsten Familienmitgliedern soll (gerade bei einem negativen Ausgang der Reanimation) umgehend gesprochen werden, und sie sollen die Möglichkeit bekommen, Fragen über Details des Verlaufs der Reanimation stellen zu können. Außerdem sollten sie über Möglichkeiten weiterer Unterstützung informiert werden [286].

In die Entscheidung, ob und wann eine Neugeborenenreanimation beendet wird, soll idealerweise ein erfahrener Kinderarzt eingebunden sein. Ob Reanimationsmaßnahmen bei extremer Frühgeburtlichkeit eingeleitet werden, soll - wann immer möglich - in enger Abstimmung zwischen Eltern, erfahrenen Kinderärzten und Geburtshelfern erfolgen. Sind bei einem Kind bereits pränatal z. B. schwere angeborene Fehlbildungen bekannt, sollen die entsprechenden Therapiemöglichkeiten und die weitere Prognose bereits vor der Geburt mit Eltern, Hebammen, Geburtshelfern und erstversorgendem Team besprochen werden [283].

Alle Gespräche und Entscheidungen müssen vor der Geburt sorgfältig im Krankenblatt der Schwangeren und postnatal im Krankenblatt des Neugeborenen dokumentiert werden.

\section{Weitere Versorgung nach erfolgreicher Reanimation (,post-resuscitation care ${ }^{\prime \prime}$ )}

Auch nach anfänglicher Stabilisierung können sich Neugeborene nach einer Reanimation im weiteren Verlauf erneut klinisch verschlechtern. Sobald Atmung und Kreislauf stabilisiert sind, muss das Neugeborene an einem Ort verbleiben oder dorthin verlegt werden, an dem eine eng- maschige Überwachung und weitere intensivmedizinische Therapie erfolgen kann.

\section{Glukose}

In einem tierexperimentellen Asphyxieund Reanimationsmodell war eine Hypoglykämie mit einem schlechten neurologischen Outcome assoziiert [287]. Neugeborene Tiere, die während eines anoxischen oder hypoxisch-ischämischen Insults hypoglykämisch waren, zeigten im Verlauf größere zerebrale Infarktareale und/oder eine geringere Überlebensrate als die Kontrollgruppe [288, 289]. Eine klinische Studie konnte nachweisen, dass eine Hypoglykämie im Rahmen einer perinatalen Asphyxie mit einem schlechten neurologischen Outcome verbunden ist [290]. Bei Erwachsenen, Kindern und extrem unreifen Neugeborenen, die intensivmedizinisch versorgt werden, ist dagegen eine Hyperglykämie ebenfalls mit einem schlechten Outcome verbunden [288292]. Eine Hyperglykämie, speziell nach einem hypoxisch-ischämischen Ereignis bei pädiatrischen Patienten, scheint allerdings nicht schädlich zu sein [293]. Dies unterstützen Erkenntnisse aus tierexperimentellen Studien, [294] manche dieser Studien scheinen sogar auf einen protektiven Effekt einer Hyperglykämie in diesen Fällen hinzuweisen [295]. Trotzdem ermöglicht die verfügbare Datenlage keine Festlegung eines Blutglukosebereichs, der mit einer geringstmöglichen Hirnschädigung nach Asphyxie und Reanimation verbunden wäre. Bei Neugeborenen, die reanimiert wurden, sollen die Blutglukosespiegel daher überwacht und im Normbereich gehalten werden.

\section{Therapeutische Hypothermie}

Reifgeborenen und nahezu reifen Neugeborenen mit moderater bis schwerer hypoxisch-ischämischer Enzephalopathie soll, wenn möglich, eine therapeutische Hypothermie geboten werden [296301]. Sowohl eine komplette Körperkühlung als auch eine selektive Kopfkühlung sind hierfür geeignete Methoden. Für den Beginn und die Durchführung einer Kühlung sollen definierte standardisierte Protokolle verwendet werden. Die Therapie erfolgt auf einer neonatologischen Intensivstation in Zusammenarbeit mit einem 
multidisziplinären Team. Die Behandlungsprotokolle sollten den in den randomisierten Studien verwendeten Studienprotokollen entsprechen (u. a. Beginn innerhalb von $6 \mathrm{~h}$ postnatal, Kühlungsdauer $72 \mathrm{~h}$, Wiedererwärmung über mindestens $4 \mathrm{~h}$ ). Tiermodelle zeigen deutlich, dass die Effektivität der Kühlung durch einen frühzeitigen Beginn günstig beeinflusst wird. Für eine Kühlung, die erst nach $6 \mathrm{~h}$ postnatal bei einem Neugeborenen begonnen wird, gibt es keine Hinweise auf Effektivität. Eine Kühlung dennoch nach der 6. Stunde zu beginnen, liegt im Ermessen des behandelnden Teams unter Berücksichtigung der individuellen Situation. Bekannte Nebenwirkungen einer therapeutischen Kühlung, wie Thrombozytopenie und Hypotension, müssen engmaschig überwacht werden. Alle mit Hypothermie behandelten Neugeborenen sollten ein Follow-up in einer neonatologischen Nachsorge erhalten.

\section{Prognostische Hilfsmittel}

Der APGAR-Score war als „einfache, gebräuchliche, eindeutige Klassifikation des Neugeborenen nach der Geburt" gedacht und sollte „als Diskussionsgrundlage und zum Vergleich verschiedener geburtshilflicher Praktiken, Verfahren zur mütterlichen Schmerztherapie und zur Beurteilung der Effektivität von Reanimationsmaßnahmen" dienen (Betonung durch die Autoren) [106]. Auch wenn der APGAR-Score weiterhin im klinischen Alltag, für wissenschaftliche Studien und als prognostisches Hilfsmittel verwendet wird [302], wird seine Eignung durch die hohe inter- und intrapersonelle Variabilität bei seiner Erhebung zunehmend infrage gestellt. Zum Teil lässt sich dies durch das fehlende Einvernehmen erklären, wie die Therapie und wie Frühgeburtlichkeit in den APGAR-Score einfließen sollen. Eine Weiterentwicklung des APGARScores soll daher in folgende Richtung erfolgen: Alle Parameter sollen anhand des tatsächlichen klinischen Zustands, unabhängig von den durchgeführten medizinischen Maßnahmen, gewertet werden, und die Bewertung soll der besonderen klinischen Situation bei Frühgeburtlichkeit Rechnung tragen. Zusätzlich sollen auch die medizinischen Maßnahmen, die zum Erreichen der klinischen Situation notwendig waren, bewertet werden. Dieser „kombinierte“ APGAR-Score scheint eine bessere prognostische Aussagekraft bei Früh- und reifen Neugeborenen zu haben $[303,304]$.

\section{Briefing/Debriefing}

Im Vorfeld einer Reanimation sollten die Zuständigkeiten der einzelnen Teammitglieder festgelegt werden. Im Anschluss an eine Neugeborenenversorgung sollen die Ereignisse in positiver und konstruktiver Weise im Team nachbesprochen werden. Gerade nach dramatischen Ereignissen soll Teammitgliedern immer auch die Möglichkeit einer psychologischen Unterstützung angeboten werden. In Studien konnte ein genereller positiver Effekt von Briefings und Debriefings auf die zukünftige Team-Performance nachgewiesen werden [305-310]. Allerdings handelte es sich bei diesen Studien häufig um Untersuchungen im Rahmen von Simulationstrainings. Versorgungen im Kreißsaal bzw. Sectio-OP zu filmen und diese nachzubesprechen, scheint ebenfalls eine sehr vielversprechende Methode für die Verbesserung der Versorgungsqualität zu sein [311]. Es konnte gezeigt werden, dass eine strukturierte Analyse des gesamten perinatologischen Managements mit Feedback zu einer Verbesserung des Outcomes und weniger intraventrikulären Blutungen bei Frühgeborenen führen [312].

Unabhängig vom Ausgang einer Reanimation kann das Miterleben der Wiederbelebung des eigenen Neugeborenen ein traumatisierendes Erlebnis für Eltern sein. Wann immer die Notwendigkeit von Reanimationsmaßnahmen im Vorfeld einer Versorgung zu erwarten ist, sollen Eltern daher darauf vorbereitet werden. Sie sollen möglichst engmaschig Informationen über den Verlauf erhalten und in jedem Fall sobald als möglich nach der Reanimation über Einzelheiten informiert werden. Idealerweise werden diese Informationen von erfahrenen Mitarbeitern übermittelt. Der frühestmögliche Kontakt zwischen Eltern und Kind hat größte Bedeutung.

\section{Korrespondenzadresse}

\section{J. Wyllie}

Abteilung für Neonatologie

The James Cook University Hospital

Middlesbrough

Jens.Schwindt@simcharacters.com

\section{Korrespondierende Übersetzer}

Dr. Jens-Christian Schwindt

Facharzt für Kinder- und Jugendheilkunde Pädiatrische Simulation und Patientensicherheit Jens.Schwindt@simcharacters.com

\section{Dr. Ulrich Kreth}

Klinik für Kinder- und Jugendmedizin Allgemeines Krankenhaus Viersen $\mathrm{GmbH}$ Hoserkirchweg 63, 41747 Viersen

kreth@akh-viersen.de

\section{Einhaltung ethischer Richtlinien}

Interessenkonflikt. C.C. Rohr erhält Lehrgelder von Fischer \& Paykel und ist medizinischer Berater für STEPHAN company; M.Rüdiger ist Ehrensprecher von Chiesi, Lyomark und erhält Forschungsgelder SLE device; J. Wyllie, B. Urlesberger, D.Trevisanuto und J. Bruinenberg geben an, dass kein Interessenkonflikt besteht

Dieser Beitrag beinhaltet keine Studien an Menschen oder Tieren.

\section{Literatur}

1. Wyllie J, Jos Bruinenberg J, Roehr CC, Rüdiger M, Trevisanuto D (2015) B. U. European Resuscitation Council Guidelines for Resuscitation 2015 Section 7 Resuscitation and Support of Transition of Babies at Birth. Resuscitation

2. Perlman JM, Wyllie J, Kattwinkel J et al (o J) Part 7: Neonatal resuscitation: 2015 International Consensus on Cardiopulmonary Resuscitation and Emergency Cardiovascular Care Science With Treatment Recommendations. Circulation (In press)

3. Richmond S, Wyllie J (2010) European Resuscitation Council Guidelines for Resuscitation 2010 Section 7. Resuscitation of babies at birth. Resuscitation 81:1389-1399

4. Wyllie J, Perlman JM, Kattwinkel J et al (2010) Part 11: Neonatal resuscitation: 2010 International Consensus on Cardiopulmonary Resuscitation and Emergency Cardiovascular Care Science with Treatment Recommendations. Resuscitation 81(Suppl 1):e260-e287

5. Ersdal HL, Mduma E, Svensen E, Perlman JM (2012) Early initiation of basic resuscitation interventions including face mask ventilation may reduce birth asphyxia related mortality in low-income countries: a prospective descriptive observational study. Resuscitation 83:869-873 
6. Perlman JM, Risser R (1995) Cardiopulmonary resuscitation in the delivery room: associated clinical events. Arch Pediatr Adolesc Med 149:20-25

7. Barber CA, Wyckoff MH (2006) Use and efficacy of endotracheal versus intravenous epinephrine during neonatal cardiopulmonary resuscitation in the delivery room. Pediatrics 118:1028-1034

8. Palme-Kilander C (1992) Methods of resuscitation in low-Apgar-score newborn infants - a national survey. Acta Paediatr 81:739-744

9. Aziz K, Chadwick M, Baker M, Andrews W (2008) Ante- and intra-partum factors that predict increased need for neonatal resuscitation. Resuscitation 79:444-452

10. Yee W, Amin H, Wood S (2008) Elective cesarean delivery, neonatal intensive care unit admission, and neonatal respiratory distress. Obstet Gynecol 111:823-828

11. Chiosi C (2013) Genetic drift. Hospital deliveries. Am J Med Genet A 161A:2122-2123

12. Ertugrul S, Gun I, Mungen E, Muhcu M, Kilic $S$ Atay V (2013) Evaluation of neonatal outcomes in elective repeat cesarean delivery at term according to weeks of gestation. J Obstet Gynaecol Res 39:105-112

13. Berthelot-Ricou A, Lacroze V, Courbiere B, Guidicelli B, Gamerre M, Simeoni U (2013) Respiratory distress syndrome after elective caesarean section in near term infants: a 5-year cohort study. J Matern Fetal Neonatal Med 26:176-182

14. Gordon A, McKechnie EJ, Jeffery H (2005) Pediatric presence at cesarean section: justified or not? Am J Obstet Gynecol 193:599-605

15. Atherton N, Parsons SJ, Mansfield P (2006) Attendance of paediatricians at elective Caesarean sections performed under regional anaesthesia: is it warranted? J Paediatr Child Health 42:332336

16. Annibale DJ, Hulsey TC, Wagner CL, Southgate WM (1995) Comparative neonatal morbidity of abdominal and vaginal deliveries after uncomplicated pregnancies. Arch Pediatr Adolesc Med 149:862-867

17. Parsons SJ, Sonneveld S, Nolan T (1998) Is a paediatrician needed at all Caesarean sections? J Paediatr Child Health 34:241-244

18. Peltonen T (1981) Placental transfusion - advantage an disadvantage. Eur J Pediatr 137:141-146

19. Brady JP, James LS (1962) Heart rate changes in the fetus and newborn infant during labor, delivery, and the immediate neonatal period. Am J Obstet Gynecol 84:1-12

20. Polglase GR, Dawson JA, Kluckow M et al (2015) Ventilation onset prior to umbilical cord clamping (physiological-based cord clamping) improves systemic and cerebral oxygenation in preterm lambs. PloS one 10:e0117504

21. Strauss RG, Mock DM, Johnson KJ et al (2008) A randomized clinical trial comparing immediate versus delayed clamping of the umbilical cord in preterm infants: short-term clinical and laboratory endpoints. Transfusion 48:658-665

22. Rabe H, Reynolds G, Diaz-Rossello J (2008) A systematic review and meta-analysis of a brief delay in clamping the umbilical cord of preterm infants. Neonatology 93:138-144

23. Ghavam S, Batra D, Mercer J et al (2014) Effects of placental transfusion in extremely low birthweight infants: meta-analysis of long- and short-term outcomes. Transfusion 54:1192-1198
24. Mercer JS, Vohr BR, McGrath MM, Padbury JF, Wallach M, Oh W (2006) Delayed cord clamping in very preterm infants reduces the incidence of intraventricular hemorrhage and late-onset sepsis: a randomized, controlled trial. Pediatrics 117:1235-1242

25. Kugelman A, Borenstein-Levin L, Riskin A et al (2007) Immediate versus delayed umbilical cord clamping in premature neonates born $<35$ weeks: a prospective, randomized, controlled study. Am J Perinatol 24:307-315

26. Katheria AC, Truong G, Cousins L, Oshiro B, Finer NN (2015) Umbilical cord milking versus delayed cord clamping in preterm infants. Pediatrics 136:61-69

27. Dahm LS, James LS (1972) Newborn temperature and calculated heat loss in the delivery room. Pediatrics 49:504-513

28. Stephenson J, Du J, TK O (1970) The effect if cooling on blood gas tensions in newborn infants. J Pediatr 76:848-852

29. Gandy GM, Adamsons K Jr, Cunningham N, Silverman WA, James LS (1964) Thermal environment and acid-base homeostasis in human infants during the first few hours of life. J Clin Invest $43: 751-758$

30. Budin P, The Nursling (1907) The feeding and hygiene of premature and full-term infants. Translation by WJ Maloney. The Caxton Publishing Company, London

31. Abd-El Hamid S, Badr-El Din MM, Dabous NI, Saad KM (2012) Effect of the use of a polyethylene wrap on the morbidity and mortality of very low birth weight infants in Alexandria University Children's Hospital. J Egypt Public Health Assoc 87:104-108

32. Acolet D, Elbourne D, Mclntosh N et al (2005) Project 27/28: inquiry into quality of neonatal care and its effect on the survival of infants who were born at 27 and 28 weeks in England, Wales, and Northern Ireland. Pediatrics 116:1457-1465

33. Bateman DA, O'Bryan L, Nicholas SW, Heagarty MC (1994) Outcome of unattended out-of-hospital births in Harlem. Arch Pediatr Adolesc Med 148:147-152

34. Bhoopalam PS, Watkinson M (1991) Babies born before arrival at hospital. Br J Obstet Gynaecol 98:57-64

35. Boo NY, Guat-Sim Cheah I, Malaysian National Neonatal Registry (2013) Admission hypothermia among VLBW infants in Malaysian NICUs. J Trop Pediatr 59:447-452

36. Buetow KC, Kelein SW (1964) Effects of maintenenance of "normal" skin temperature on survival of infants of low birth weight. Pediatrics 33:163-169

37. Costeloe K, Hennessy E, Gibson AT, Marlow N, Wilkinson AR (2000) The EPICure study: outcomes to discharge from hospital for infants born at the threshold of viability. Pediatrics 106:659671

38. Costeloe KL, Hennessy EM, Haider S, Stacey F, Marlow N, Draper ES (2012) Short term outcomes after extreme preterm birth in England: comparison of two birth cohorts in 1995 and 2006 (the EPICure studies). BMJ 345:e7976

39. da Mota Silveira SM, Goncalves de Mello MJ, de Arruda Vidal S, de Frias PG, Cattaneo A (2003) Hypothermia on admission: a risk factor for death in newborns referred to the Pernambuco Institute of Mother and Child Health. J Trop Pediatr 49:115-120
40. Daga AS, Daga SR, Patole SK (1991) Determinants of death among admissions to intensive care unit for newborns. J Trop Pediatr 37:53-56

41. de Almeida MF, Guinsburg R, Sancho GA et al (2014) Hypothermia and early neonatal mortality in preterm infants. J Pediatr 164:271-275e1

42. Garcia-Munoz Rodrigo F, Rivero Rodriguez S, Siles Quesada C (2014) [Hypothermia risk factors in the very low weight newborn and associated morbidity and mortality in a neonatal care unit]. An Pediatr (Barc) 80:144-150

43. Harms K, Osmers R, Kron M et al (1994) [Mortality of premature infants 1980-1990: analysis of data from the Gottingen perinatal center]. Z Geburtshilfe Perinatol 198:126-133

44. Hazan J, Maag U, Chessex P (1991) Association between hypothermia and mortality rate of premature infants - revisited. Am J Obstet Gynecol 164:111-112

45. Jones $P$, Alberti C, Jule L et al (2011) Mortality in out-of-hospital premature births. Acta Paediatr 100:181-187

46. Kalimba E, Ballot D (2013) Survival of extremely low-birth-weight infants. S Afr J Child Health 7(1):13-16

47. Kambarami R, Chidede O (2003) Neonatal hypothermia levels and risk factors for mortality in a tropical country. Cent Afr J Med 49:103-106

48. Kent AL, Williams J (2008) Increasing ambient operating theatre temperature and wrapping in polyethylene improves admission temperature in premature infants. J Paediatr Child Health 44:325-331

49. Laptook AR, Salhab W, Bhaskar B, Neonatal Research Network (2007) Admission temperature of low birth weight infants: predictors and associated morbidities. Pediatrics 119:e643-e649

50. Lee HC, Ho QT, Rhine WD (2008) A quality improvement project to improve admission temperatures in very low birth weight infants. J Perinatol 28:754-758

51. Levi S, Taylor W, Robinson LE, Levy LI (1984) Analysis of morbidity and outcome of infants weighing less than 800 grams at birth. South Med J 77:975-978

52. Manani $M$, Jegatheesan $P$, DeSandre G, Song $D$, Showalter L, Govindaswami B (2013) Elimination of admission hypothermia in preterm very lowbirth-weight infants by standardization of delivery room management. Perm J 17:8-13

53. Manji KP, Kisenge R (2003) Neonatal hypothermia on admission to a special care unit in Dar-esSalaam, Tanzania: a cause for concern. Cent Afr J Med 49:23-27

54. Mathur NB, Krishnamurthy S, Mishra TK (2005) Evaluation of WHO classification of hypothermia in sick extramural neonates as predictor of fatality. J Trop Pediatr 51:341-345

55. Miller SS, Lee HC, Gould JB (2011) Hypothermia in very low birth weight infants: distribution, risk factors and outcomes. J Perinatol 31(Suppl 1):S49-S56

56. Mullany LC, Katz J, Khatry SK, LeClerq SC, Darmstadt GL, Tielsch JM (2010) Risk of mortality associated with neonatal hypothermia in southern Nepal. Arch Pediatr Adolesc Med 164:650-656

57. Nayeri F, Nili F (2006) Hypothermia at birth and its associated complications in newborn infants: a follow up study. Iranian J Public Health 35(1):48-52

58. Obladen M, Heemann U, Hennecke KH, Hanssler $L$ (1985) [Causes of neonatal mortality 19811983: a regional analysis]. Z Geburtshilfe Perinatol 189:181-187 
59. Ogunlesi TA, Ogunfowora OB, Adekanmbi FA, Fetuga BM, Olanrewaju DM (2008) Point-of-admission hypothermia among high-risk Nigerian newborns. BMC Pediatr 8:40

60. Pal DK, Manandhar DS, Rajbhandari S, Land JM, Patel N, de L Costello AM (2000) Neonatal hypoglycaemia in Nepal 1. Prevalence and risk factors. Arch Dis Child Fetal Neonatal Ed 82:F46-F51

61. Shah S, Zemichael O, Meng HD (2012) Factors associated with mortality and length of stay in hospitalised neonates in Eritrea, Africa: a cross-sectional study. BMJ Open 2. doi:10.1136/bmjopen-2011-000792

62. Singh A, Yadav A, Singh A (2012) Utilization of postnatal care for newborns and its association with neonatal mortality in India: an analytical appraisal. BMC Pregnancy Childbirth 12:33

63. Sodemann M, Nielsen J, Veirum J, Jakobsen MS, Biai S, Aaby P (2008) Hypothermia of newborns is associated with excess mortality in the first 2 months of life in Guinea-Bissau, West Africa. Trop Med Int Health 13:980-986

64. Stanley FJ, Alberman EV (1978) Infants of very low birthweight. I: perinatal factors affecting survival. Dev Med Child Neurol 20:300-312

65. Wyckoff MH, Perlman JM (2004) Effective ventilation and temperature control are vital to outborn resuscitation. Prehosp Emerg Care 8:191-195

66. Bartels DB, Kreienbrock L, Dammann O, Wenzlaff P, Poets CF (2005) Population based study on the outcome of small for gestational age newborns. Arch Dis Childh Fetal Neonatal Ed 90:F53-F59

67. Carroll PD, Nankervis CA, Giannone PJ, Cordero $L$ (2010) Use of polyethylene bags in extremely low birth weight infant resuscitation for the prevention of hypothermia. J Reprod Med 55:9-13

68. Gleissner M, Jorch G, Avenarius S (2000) Risk factors for intraventricular hemorrhage in a birth cohort of 3721 premature infants. J Perinat Med 28:104-110

69. Herting E, Speer CP, Harms K et al (1992) Factors influencing morbidity and mortality in infants with severe respiratory distress syndrome treated with single or multiple doses of a natural porcine surfactant. Biol Neonate 61(Suppl 1):26-30

70. DeMauro SB, Douglas E, Karp K et al (2013) Improving delivery room management for very preterm infants. Pediatrics 132:e1018-e1025

71. Harms K, Herting E, Kron M, Schill M, Schiffmann $H$ (1997) [Importance of pre- and perinatal risk factors in respiratory distress syndrome of premature infants. A logical regression analysis of 1100 cases]. Z Geburtshilfe Neonatol 201:258262

72. Lee HC, Powers RJ, Bennett MV et al (2014) Implementation methods for delivery room management: a quality improvement comparison study. Pediatrics 134:e1378-e1386

73. Reilly MC, Vohra S, Rac VE et al (2015) Randomized trial of occlusive wrap for heat loss prevention in preterm infants. J Pediatr 166:262-268e2

74. Zayeri F, Kazemnejad A, Ganjali M, Babaei G, Khanafshar N, Nayeri F (2005) Hypothermia in Iranian newborns. Incidence, risk factors and related complications. Saudi Med J 26:1367-1371

75. Anderson S, Shakya KN, Shrestha LN, Costello AM (1993) Hypoglycaemia: a common problem among uncomplicated newborn infants in Nepal. J Trop Pediatr 39:273-277

76. Lazic-Mitrovic T, Djukic M, Cutura N et al (2010) [Transitory hypothermia as early prognostic factor in term newborns with intrauterine growth retardation]. Srp Arh Celok Lek 138:604-608
77. Lenclen R, Mazraani M, Jugie M et al (2002) [Use of a polyethylene bag: a way to improve the thermal environment of the premature newborn at the delivery room]. Arch Pediatr 9:238-244

78. Sasidharan CK, Gokul E, Sabitha S (2004) Incidence and risk factors for neonatal hypoglycaemia in Kerala, India. Ceylon Med J 49:110-113

79. Mullany LC (2010) Neonatal hypothermia in lowresource settings. Semin Perinatol 34:426-433

80. World Health Organization (1997) Department of Reproductive Health and Research (RHR) Thermal protection of the newborn: a practical guide (WHO/RHT/MSM/97.2) Geneva

81. Dahm LS, James LS (1972) Newborn temperature and calculated heat loss in the delivery room. Pediatrics 49:504-513

82. Vohra S, Frent G, Campbell V, Abbott M, Whyte $R$ (1999) Effect of polyethylene occlusive skin wrapping on heat loss in very low birth weight infants at delivery: a randomized trial. J Pediatr 134:547-551

83. Bjorklund LJ, Hellstrom-Westas L (2000) Reducing heat loss at birth in very preterm infants. J Pediatr 137:739-740

84. Meyer MP, Payton MJ, Salmon A, Hutchinson C, de Klerk A (2001) A clinical comparison of radiant warmer and incubator care for preterm infants from birth to 1800 grams. Pediatrics 108:395401

85. te Pas AB, Lopriore E, Dito I, Morley CJ, Walther FJ (2010) Humidified and heated air during stabilization at birth improves temperature in preterm infants. Pediatrics 125:e1427-e1432

86. Russo A, McCready M, Torres L et al (2014) Reducing hypothermia in preterm infants following delivery. Pediatrics 133:e1055-e1062

87. Pinheiro JM, Furdon SA, Boynton S, Dugan R, Reu-Donlon C, Jensen S (2014) Decreasing hypothermia during delivery room stabilization of preterm neonates. Pediatrics 133:e218-e226

88. McCarthy LK, Molloy EJ, Twomey AR, Murphy JF, O'Donnell CP (2013) A randomized trial of exothermic mattresses for preterm newborns in polyethylene bags. Pediatrics 132:e135-e141

89. Billimoria Z, Chawla S, Bajaj M, Natarajan G (2013) Improving admission temperature in extremely low birth weight infants: a hospital-based multi-intervention quality improvement project. J Perinat Med 41:455-460

90. Chawla S, Amaram A, Gopal SP, Natarajan G (2011) Safety and efficacy of Trans-warmer mattress for preterm neonates: results of a randomized controlled trial. J Perinatol 31:780-784

91. Ibrahim CP, Yoxall CW (2010) Use of self-heating gel mattresses eliminates admission hypothermia in infants born below 28 weeks gestation. Eur J Pediatr 169:795-799

92. Singh A, Duckett J, Newton T, Watkinson M (2010) Improving neonatal unit admission temperatures in preterm babies: exothermic mattresses, polythene bags or a traditional approach? J Perinatol 30:45-49

93. Belsches TC, Tilly AE, Miller TR et al (2013) Randomized trial of plastic bags to prevent term neonatal hypothermia in a resource-poor setting. Pediatrics 132:e656-e661

94. Leadford AE, Warren JB, Manasyan A et al (2013) Plastic bags for prevention of hypothermia in preterm and low birth weight infants. Pediatrics 132:e128-e134
95. Bergman NJ, Linley LL, Fawcus SR (2004) Randomized controlled trial of skin-to-skin contact from birth versus conventional incubator for physiological stabilization in 1200- to 2199-gram newborns. Acta paediatrica 93:779-785

96. Fardig JA (1980) A comparison of skin-to-skin contact and radiant heaters in promoting neonatal thermoregulation. J Nurse Midwifery 25:1928

97. Christensson K, Siles C, Moreno L et al (1992) Temperature, metabolic adaptation and crying in healthy full-term newborns cared for skin-to-skin or in a cot. Acta paediatr 81:488-493

98. Christensson K (1996) Fathers can effectively achieve heat conservation in healthy newborn infants. Acta Paediatr 85:1354-1360

99. Bystrova K, Widstrom AM, Matthiesen AS et al (2003) Skin-to-skin contact may reduce negative consequences of "the stress of being born": a study on temperature in newborn infants, subjected to different ward routines in St. Petersburg. Acta Paediatr 92:320-326

100. Nimbalkar SM, Patel VK, Patel DV, Nimbalkar AS, Sethi A, Phatak A (2014) Effect of early skin-toskin contact following normal delivery on incidence of hypothermia in neonates more than $1800 \mathrm{~g}$ : randomized control trial. J Perinatol 34:364-368

101. Marin Gabriel MA, Llana Martin I, Lopez Escobar A, Fernandez Villalba E, Romero Blanco I, Touza Pol P (2010) Randomized controlled trial of early skin-to-skin contact: effects on the mother and the newborn. Acta Paediatr 99:1630-1634

102. Lieberman $E$, Eichenwald $E$, Mathur $G$, Richardson D, Heffner L, Cohen A (2000) Intrapartum fever and unexplained seizures in term infants. Pediatrics 106:983-988

103. Grether JK, Nelson KB (1997) Maternal infection and cerebral palsy in infants of normal birth weight. JAMA 278:207-211

104. Coimbra C, Boris-Moller F, Drake M, Wieloch T (1996) Diminished neuronal damage in the rat brain by late treatment with the antipyretic drug dipyrone or cooling following cerebral ischemia. Acta Neuropathol 92:447-453

105. Dietrich WD, Alonso O, Halley M, Busto R (1996) Delayed posttraumatic brain hyperthermia worsens outcome after fluid percussion brain injury: a light and electron microscopic study in rats. Neurosurgery 38:533-541. (discussion 41)

106. Apgar V (1953) A proposal for a new method of evaluation of the newborn infant. Curr Res Anesth Analg 32:260-267

107. Chamberlain G, Banks J (1974) Assessment of the Apgar score. Lancet 2:1225-1228

108. Owen CJ, Wyllie JP (2004) Determination of heart rate in the baby at birth. Resuscitation 60:213217

109. Kamlin CO, O'Donnell CP, Everest NJ, Davis PG, Morley CJ (2006) Accuracy of clinical assessment of infant heart rate in the delivery room. Resuscitation 71:319-321

110. Dawson JA, Saraswat A, Simionato L et al (2013) Comparison of heart rate and oxygen saturation measurements from Masimo and Nellcor pulse oximeters in newly born term infants. Acta Paediatr 102:955-960

111. Kamlin CO, Dawson JA, O'Donnell CP et al (2008) Accuracy of pulse oximetry measurement of heart rate of newborn infants in the delivery room. J Pediatr 152:756-760 
112. Katheria A, Rich W, Finer N (2012) Electrocardiogram provides a continuous heart rate faster than oximetry during neonatal resuscitation. Pediatrics 130:e1177-1181

113. Voogdt KG, Morrison AC, Wood FE, van Elburg RM, Wyllie JP (2010) A randomised, simulated study assessing auscultation of heart rate at birth. Resuscitation 81:1000-1003

114. Mizumoto H, Tomotaki S, Shibata H et al (2012) Electrocardiogram shows reliable heart rates much earlier than pulse oximetry during neonatal resuscitation. Pediatr Int 54:205-207

115. van Vonderen JJ, Hooper SB, Kroese JK et al (2015) Pulse oximetry measures a lower heart rate at birth compared with electrocardiography. J Pediatr 166:49-53

116. O'Donnell CP, Kamlin CO, Davis PG, Carlin JB, Morley CJ (2007) Clinical assessment of infant colour at delivery. Arch Dis Child Fetal Neonatal Ed 92:F465-F467

117. Konstantelos $D$, Gurth $H$, Bergert $R$, Ifflaender $S$, Rudiger M (2014) Positioning of term infants during delivery room routine handling - analysis of videos. BMC Pediatr 14:33

118. Kelleher J, Bhat R, Salas AA et al (2013) Oronasopharyngeal suction versus wiping of the mouth and nose at birth: a randomised equivalency trial. Lancet 382:326-330

119. Cordero L Jr, Hon EH (1971) Neonatal bradycardia following nasopharyngeal stimulation. J Pediatr 78:441-447

120. Gungor S, Kurt E, Teksoz E, Goktolga U, Ceyhan T, Baser I (2006) Oronasopharyngeal suction versus no suction in normal and term infants delivered by elective cesarean section: a prospective randomized controlled trial. Gynecol Obstet Invest 61:9-14

121. Waltman PA, Brewer JM, Rogers BP, May WL (2004) Building evidence for practice: a pilot study of newborn bulb suctioning at birth. J Midwifery Womens Health 49:32-38

122. Carson BS, Losey RW, Bowes WA Jr, Simmons MA (1976) Combined obstetric and pediatric approach to prevent meconium aspiration syndrome. Am J Obstet Gynecol 126:712-715

123. Ting P, Brady JP (1975) Tracheal suction in meconium aspiration. Am J Obstet Gynecol 122:767771

124. Falciglia HS, Henderschott $C$, Potter $P$, Helmchen $R$ (1992) Does DeLee suction at the perineum prevent meconium aspiration syndrome? Am J Obstet Gynecol 167:1243-1249

125. Wiswell TE, Tuggle JM, Turner BS (1990) Meconium aspiration syndrome: have we made a difference? Pediatrics 85:715-721

126. Wiswell TE, Gannon CM, Jacob J et al (2000) Delivery room management of the apparently vigorous meconium-stained neonate: results of the multicenter, international collaborative trial. Pediatrics 105:1-7

127. Vain NE, Szyld EG, Prudent LM, Wiswell TE, Aguilar AM, Vivas NI (2004) Oropharyngeal and nasopharyngeal suctioning of meconium-stained neonates before delivery of their shoulders: multicentre, randomised controlled trial. Lancet 364:597-602

128. Chettri S, Adhisivam B, Bhat BV (2015) Endotracheal suction for nonvigorous neonates born through meconium stained amniotic fluid: a randomized controlled trial. J Pediatr. doi:10.1016/j. jpeds.2014.12.076
129. Al Takroni AM, Parvathi CK, Mendis KB, Hassan S, Reddy I, Kudair HA (1998) Selective tracheal suctioning to prevent meconium aspiration syndrome. Int J Gynaecol Obstet 63:259-263

130. Davis RO, Philips JB 3rd, Harris BA Jr, Wilson ER, Huddleston JF (1985) Fatal meconium aspiration syndrome occurring despite airway management considered appropriate. Am J Obstet Gynecol 151:731-736

131. Manganaro R, Mami C, Palmara A, Paolata A, Gemelli $M$ (2001) Incidence of meconium aspiration syndrome in term meconium-stained babies managed at birth with selective tracheal intubation. J Perinat Med 29:465-468

132. Yoder BA (1994) Meconium-stained amniotic fluid and respiratory complications: impact of selective tracheal suction. Obstet Gynecol 83:77-84

133. Bent RC, Wiswell TE, Chang A (1992) Removing meconium from infant tracheae. What works best? Am J Dis Child 146:1085-1089

134. Dargaville PA, Copnell B, Mills JF et al (2011) Randomized controlled trial of lung lavage with dilute surfactant for meconium aspiration syndrome. J Pediatr 158:383-389e2

135. Dargaville PA, Copnell B, Mills JF et al (2013) Fluid recovery during lung lavage in meconium aspiration syndrome. Acta Paediatr 102:e90-e93

136. Wyllie J, Perlman JM, Kattwinkel J et al (2010) Part 11: Neonatal resuscitation: 2010 International Consensus on Cardiopulmonary Resuscitation and Emergency Cardiovascular Care Science with Treatment Recommendations. Resuscitation 81(Suppl 1):e260-e287

137. Vyas H, Milner AD, Hopkin IE, Boon AW (1981) Physiologic responses to prolonged and slow-rise inflation in the resuscitation of the asphyxiated newborn infant. J Pediatr 99:635-639

138. Mortola JP, Fisher JT, Smith JB, Fox GS, Weeks S, Willis D (1982) Onset of respiration in infants delivered by cesarean section. J Appl Physiol 52:716724

139. Hull D (1969) Lung expansion and ventilation during resuscitation of asphyxiated newborn infants. J Pediatr 75:47-58

140. Vyas H, Milner AD, Hopkins IE (1981) Intrathoracic pressure and volume changes during the spontaneous onset of respiration in babies born by cesarean section and by vaginal delivery. J Pediatr 99:787-791

141. Vyas H, Field D, Milner AD, Hopkin IE (1986) Determinants of the first inspiratory volume and functional residual capacity at birth. Pediatr Pulmonol 2:189-193

142. Boon AW, Milner AD, Hopkin IE (1979) Lung expansion, tidal exchange, and formation of the functional residual capacity during resuscitation of asphyxiated neonates. J Pediatr 95:1031-1036

143. Hird MF, Greenough A, Gamsu HR (1991) Inflating pressures for effective resuscitation of preterm infants. Early Hum Dev 26:69-72

144. Lindner W, Vossbeck S, Hummler H, Pohlandt F (1999) Delivery room management of extremely low birth weight infants: spontaneous breathing or intubation? Pediatrics 103:961-967

145. Wood FE, Morley CJ, Dawson JA et al (2008) Assessing the effectiveness of two round neonatal resuscitation masks: study 1. Arch Dis Child Fetal Neonatal Ed 93:F235-F237

146. Wood FE, Morley CJ, Dawson JA et al (2008) Improved techniques reduce face mask leak during simulated neonatal resuscitation: study 2 . Arch Dis Child Fetal Neonatal Ed 93:F230-F234
147. Tracy MB, Klimek J, Coughtrey H et al (2011) Mask leak in one-person mask ventilation compared to two-person in newborn infant manikin study. Arch Dis Child Fetal Neonatal Ed 96:F195F200

148. Schmolzer GM, Dawson JA, Kamlin CO, O'Donnell CP, Morley CJ, Davis PG (2011) Airway obstruction and gas leak during mask ventilation of preterm infants in the delivery room. Arch Dis Child Fetal Neonatal Ed 96:F254-F257

149. Schmolzer GM, Kamlin OC, O'Donnell CP, Dawson JA, Morley CJ, Davis PG (2010) Assessment of tidal volume and gas leak during mask ventilation of preterm infants in the delivery room. Arch Dis Child Fetal Neonatal Ed 95:F393-F397

150. Klingenberg C, Sobotka KS, Ong T et al (2013) Effect of sustained inflation duration; resuscitation of near-term asphyxiated lambs. Arch Dis Child Fetal Neonatal Ed 98:F222-F227

151. te Pas AB, Siew M, Wallace MJ et al (2009) Effect of sustained inflation length on establishing functional residual capacity at birth in ventilated premature rabbits. Pediatr Res 66:295-300

152. Harling AE, Beresford MW, Vince GS, Bates M, Yoxall CW (2005) Does sustained lung inflation at resuscitation reduce lung injury in the preterm infant? Arch Dis Child Fetal Neonatal Ed 90:F406F410

153. Lindner W, Hogel J, Pohlandt F (2005) Sustained pressure-controlled inflation or intermittent mandatory ventilation in preterm infants in the delivery room? A randomized, controlled trial on initial respiratory support via nasopharyngeal tube. Acta Paediatr 94:303-309

154. Lista G, Boni L, Scopesi F et al (2015) Sustained lung inflation at birth for preterm infants: a randomized clinical trial. Pediatrics 135:e457-e464

155. Lista G, Fontana P, Castoldi F, Cavigioli F, Dani C (2011) Does sustained lung inflation at birth improve outcome of preterm infants at risk for respiratory distress syndrome? Neonatology 99:45-50

156. Mariani G, Dik PB, Ezquer A et al (2007) Pre-ductal and post-ductal $\mathrm{O} 2$ saturation in healthy term neonates after birth. J Pediatr 150:418-421

157. Dawson JA, Kamlin CO, Vento M et al (2010) Defining the reference range for oxygen saturation for infants after birth. Pediatrics 125:e1340 e1347

158. Davis PG, Tan A, O'Donnell CP, Schulze A (2004) Resuscitation of newborn infants with $100 \%$ oxygen or air: a systematic review and meta-analysis. Lancet 364:1329-1333

159. Vento M, Moro M, Escrig R et al (2009) Preterm resuscitation with low oxygen causes less oxidative stress, inflammation, and chronic lung disease. Pediatrics. doi:10.1542/peds.2009-0434

160. Saugstad OD, Aune D, Aguar M, Kapadia V, Finer N, Vento M (2014) Systematic review and metaanalysis of optimal initial fraction of oxygen levels in the delivery room at $\leq 32$ weeks. Acta Paediatr 103:744-751

161. Armanian AM, Badiee Z (2012) Resuscitation of preterm newborns with low concentration oxygen versus high concentration oxygen. J Res Pharm Pract 1:25-29

162. Kapadia VS, Chalak LF, Sparks JE, Allen JR, Savani RC, Wyckoff MH (2013) Resuscitation of preterm neonates with limited versus high oxygen strategy. Pediatrics 132:e1488-e1496

163. Lundstrom KE, Pryds O, Greisen G (1995) Oxygen at birth and prolonged cerebral vasoconstriction in preterm infants. Arch Dis Child Fetal Neonatal Ed 73:F81-F86 
164. Rabi Y, Singhal N, Nettel-Aguirre A (2011) Roomair versus oxygen administration for resuscitation of preterm infants: the ROAR study. Pediatrics 128:e374-e381

165. Rook D, Schierbeek H, Vento M et al (2014) Resuscitation of preterm infants with different inspired oxygen fractions. J Pediatr 164:1322-1326e3

166. Wang CL, Anderson C, Leone TA, Rich W, Govindaswami B, Finer NN (2008) Resuscitation of preterm neonates by using room air or $100 \%$ oxygen. Pediatrics 121:1083-1089

167. O'Donnell CP, Kamlin CO, Davis PG, Morley CJ (2005) Feasibility of and delay in obtaining pulse oximetry during neonatal resuscitation. J Pediatr 147:698-699

168. Dawson JA, Kamlin CO, Wong C et al (2009) Oxygen saturation and heart rate during delivery room resuscitation of infants $<30$ weeks' gestation with air or $100 \%$ oxygen. Arch Dis Child Fetal Neonatal Ed 94:F87-F91

169. Dildy GA, van den Berg PP, Katz M et al (1994) Intrapartum fetal pulse oximetry: fetal oxygen saturation trends during labor and relation to delivery outcome. Am J Obstet Gynecol 171:679-684

170. Rabi Y, Yee W, Chen SY, Singhal N (2006) Oxygen saturation trends immediately after birth. J Pediatr 148:590-594

171. Gonzales GF, Salirrosas A (2005) Arterial oxygen saturation in healthy newborns delivered at term in Cerro de Pasco (4340 m) and Lima (150 m). Reprod Biol Endocrinol 3:46

172. Smit M, Dawson JA, Ganzeboom A, Hooper SB van Roosmalen J, te Pas $A B$ (2014) Pulse oximetry in newborns with delayed cord clamping and immediate skin-to-skin contact. Arch Dis Child Fetal Neonatal Ed 99:F309-F314

173. Wyllie J, Perlman JM, Kattwinkel J et al (2015) Part 7: Neonatal resuscitation: 2015 International consensus on cardiopulmonary resuscitation and emergency cardiovascular care science with treatment recommendations. Resuscitation

174. Ingimarsson J, Bjorklund $\mathrm{L}$, Curstedt $\mathrm{T}$ et al (2004) Incomplete protection by prophylactic surfactant against the adverse effects of large lung inflations at birth in immature lambs. Intensive Care Med 30:1446-1453

175. Muscedere JG, Mullen JB, Gan K, Slutsky AS (1994) Tidal ventilation at low airway pressures can augment lung injury. Am J Respir Crit Care Med 149:1327-1334

176. Naik AS, Kallapur SG, Bachurski CJ et al (2001) Effects of ventilation with different positive endexpiratory pressures on cytokine expression in the preterm lamb lung. Am J Respir Crit Care Med 164:494-498

177. Polglase GR, Hillman NH, Pillow JJ et al (2008) Positive end-expiratory pressure and tidal volume during initial ventilation of preterm lambs. Pediatr Res 64:517-522

178. Nilsson R, Grossmann G, Robertson B (1980) Bronchiolar epithelial lesions induced in the premature rabbit neonate by short periods of artificial ventilation. Acta Pathol Microbiol Scand 88:359-367

179. Probyn ME, Hooper SB, Dargaville PA et al (2004) Positive end expiratory pressure during resuscitation of premature lambs rapidly improves blood gases without adversely affecting arterial pressure. Pediatr Res 56:198-204

180. te Pas AB, Siew M, Wallace MJ et al (2009) Establishing functional residual capacity at birth: the effect of sustained inflation and positive end-expiratory pressure in a preterm rabbit model. Pediatr Res 65:537-541
181. Dawson JA, Schmolzer GM, Kamlin CO et al (2011) Oxygenation with T-piece versus self-inflating bag for ventilation of extremely preterm infants at birth: a randomized controlled trial. J Pediatr 158:912-918 e1-2

182. Szyld E, Aguilar A, Musante GA et al (2014) Comparison of devices for newborn ventilation in the delivery room. J Pediatr 165:234-239e3

183. Allwood AC, Madar RJ, Baumer JH, Readdy L, Wright $D$ (2003) Changes in resuscitation practice at birth. Arch Dis Child Fetal Neonatal Ed 88:F375-F379

184. Cole AF, Rolbin SH, Hew EM, Pynn S (1979) An improved ventilator system for delivery-room management of the newborn. Anesthesiology 51:356-358

185. Hoskyns EW, Milner AD, Hopkin IE (1987) A simple method of face mask resuscitation at birth. Arch Dis Child 62:376-378

186. Ganga-Zandzou PS, Diependaele JF, Storme L et al (1996) [ls Ambu ventilation of newborn infants a simple question of finger-touch?]. Arch Pediatr 3:1270-1272

187. Oddie S, Wyllie J, Scally A (2005) Use of self-inflating bags for neonatal resuscitation. Resuscitation 67:109-112

188. Finer NN, Rich W, Craft A, Henderson C (2001) Comparison of methods of bag and mask ventilation for neonatal resuscitation. Resuscitation 49:299-305

189. Dawson JA, Gerber A, Kamlin CO, Davis PG, Morley CJ (2011) Providing PEEP during neonatal resuscitation: which device is best? J Paediatr Child Health 47:698-703

190. Roehr CC, Kelm M, Fischer HS, Buhrer C, Schmalisch G, Proquitte H (2010) Manual ventilation devices in neonatal resuscitation: tidal volume and positive pressure-provision. Resuscitation 81:202-205

191. Kanter RK (1987) Evaluation of mask-bag ventilation in resuscitation of infants. Am J Dis Child 141:761-763

192. Morley CJ, Dawson JA, Stewart MJ, Hussain F, Davis PG (2010) The effect of a PEEP valve on a Laerdal neonatal self-inflating resuscitation bag. J Paediatr Child Health 46:51-56

193. Bennett S, Finer NN, Rich W, Vaucher Y (2005) A comparison of three neonatal resuscitation devices. Resuscitation 67:113-118

194. Kelm M, Proquitte H, Schmalisch G, Roehr CC (2009) Reliability of two common PEEP-generating devices used in neonatal resuscitation. Klin Padiatr 221:415-418

195. Hartung JC, Schmolzer G, Schmalisch G, Roehr CC (2013) Repeated thermo-sterilisation further affects the reliability of positive end-expiratory pressure valves. J Paediatr Child Health 49:741745

196. Schmolzer GM, Morley CJ, Wong C et al (2012) Respiratory function monitor guidance of mask ventilation in the delivery room: a feasibility study. J Pediatr 160:377-381e2

197. Kong JY, Rich W, Finer NN, Leone TA (2013) Quantitative end-tidal carbon dioxide monitoring in the delivery room: a randomized controlled trial. J Pediatr 163:104-108e1

198. Leone TA, Lange A, Rich W, Finer NN (2006) Disposable colorimetric carbon dioxide detector use as an indicator of a patent airway during noninvasive mask ventilation. Pediatrics 118:e202e204
199. McCarthy LK, Twomey AR, Molloy EJ, Murphy JF, O'Donnell CP (2013) A randomized trial of nasal prong or face mask for respiratory support for preterm newborns. Pediatrics 132:e389-e395

200. Kamlin CO, Schilleman K, Dawson JA et al (2013) Mask versus nasal tube for stabilization of preterm infants at birth: a randomized controlled trial. Pediatrics 132:e381-e388

201. Trevisanuto D, Cavallin F, Nguyen LN et al (2015) Supreme laryngeal mask airway versus face mask during neonatal resuscitation: a randomized controlled trial. J Pediatr 167(2):286-291.e1

202. Esmail N, Saleh M et al (2002) Laryngeal mask airway versus endotracheal intubation for Apgar score improvement in neonatal resuscitation. Egyptian J Anesthesiol 18:115-121

203. Trevisanuto D, Micaglio M, Pitton M, Magarotto M, Piva D, Zanardo V (2004) Laryngeal mask airway: is the management of neonates requiring positive pressure ventilation at birth changing? Resuscitation 62:151-157

204. Singh R (2005) Controlled trial to evaluate the use of LMA for neonatal resuscitation. J Anaesth Clin Pharmacol 21:303-306

205. Zhu XY, Lin BC, Zhang QS, Ye HM, Yu RJ (2011) A prospective evaluation of the efficacy of the laryngeal mask airway during neonatal resuscitation. Resuscitation 82:1405-1409

206. Schmolzer GM, Agarwal M, Kamlin CO, Davis PG (2013) Supraglottic airway devices during neonatal resuscitation: an historical perspective, systematic review and meta-analysis of available clinical trials. Resuscitation 84:722-730

207. Kempley ST, Moreiras JW, Petrone FL (2008) Endotracheal tube length for neonatal intubation. Resuscitation 77:369-373

208. Gill I, O'Donnell CP (2014) Vocal cord guides on neonatal endotracheal tubes. Arch Dis Child Fetal Neonatal Ed 99:F344

209. Palme-Kilander C, Tunell R (1993) Pulmonary gas exchange during facemask ventilation immediately after birth. Arch Dis Child 68:11-16

210. Aziz HF, Martin JB, Moore JJ (1999) The pediatric disposable end-tidal carbon dioxide detector role in endotracheal intubation in newborns. J Perinatol 19:110-113

211. Bhende MS, LaCovey D (1995) A note of caution about the continuous use of colorimetric end-tidal $\mathrm{CO}_{2}$ detectors in children. Pediatrics 95:800801

212. Repetto JE, Donohue P-CP, Baker SF, Kelly L, Nogee LM (2001)Use of capnography in the delivery room for assessment of endotracheal tube placement. J Perinatol 21:284-287

213. Roberts WA, Maniscalco WM, Cohen AR, Litman RS, Chhibber A (1995) The use of capnography for recognition of esophageal intubation in the neonatal intensive care unit. Pediatr Pulmonol 19:262-268

214. Hosono S, Inami I, Fujita H, Minato M, Takahashi $\mathrm{S}$, Mugishima H (2009) A role of end-tidal CO(2) monitoring for assessment of tracheal intubations in very low birth weight infants during neonatal resuscitation at birth. J Perinat Med 37:7984

215. Garey DM, Ward R, Rich W, Heldt G, Leone T, Finer NN (2008) Tidal volume threshold for colorimetric carbon dioxide detectors available for use in neonates. Pediatrics 121:e1524-e1527

216. Morley CJ, Davis PG, Doyle LW, Brion LP, Hascoet JM, Carlin JB (2008) Nasal CPAP or intubation at birth for very preterm infants. $\mathrm{N}$ Engl J Med 358:700-708 
217. Network SSGotEKSNNR, Finer NN, Carlo WA et al (2010) Early CPAP versus surfactant in extremely preterm infants. The New England journal of medicine 362:1970-1909

218. Dunn MS, Kaempf J, de Klerk A et al (2011) Randomized trial comparing 3 approaches to the initial respiratory management of preterm neonates. Pediatrics 128:e1069-e1076

219. Hishikawa K, Goishi K, Fujiwara T, Kaneshige M, Ito $Y$, Sago H (2015) Pulmonary air leak associated with CPAP at term birth resuscitation. Arch Dis Child Fetal Neonatal Ed

220. Poets CF, Rudiger M (2015) Mask CPAP during neonatal transition: too much of a good thing for some term infants? Arch Dis Child Fetal Neonatal Ed

221. Houri PK, Frank LR, Menegazzi JJ, Taylor R (1997) A randomized, controlled trial of two-thumb vs two-finger chest compression in a swine infant model of cardiac arrest [see comment]. Prehosp Emerg Care 1:65-67

222. David R (1988) Closed chest cardiac massage in the newborn infant. Pediatrics 81:552-554

223. Menegazzi JJ, Auble TE, Nicklas KA, Hosack GM, Rack L, Goode JS (1993) Two-thumb versus twofinger chest compression during CRP in a swine infant model of cardiac arrest. Ann Emerg Med 22:240-243

224. Thaler MM, Stobie GH (1963) An improved technique of external caridac compression in infants and young children. N Engl J Med 269:606-610

225. Christman C, Hemway RJ, Wyckoff MH, Perlman JM (2011) The two-thumb is superior to the twofinger method for administering chest compressions in a manikin model of neonatal resuscitation. Arch Dis Child Fetal Neonatal Ed 96:F99-F101

226. Dellimore K, Heunis S, Gohier F et al (2013) Development of a diagnostic glove for unobtrusive measurement of chest compression force and depth during neonatal CPR. Conf Proc IEEE Eng Med Biol Soc 2013:350-353

227. Dorfsman ML, Menegazzi JJ, Wadas RJ, Auble TE (2000) Two-thumb vs two-finger chest compression in an infant model of prolonged cardiopulmonary resuscitation. Acad Emerg Med 7:10771082

228. Martin PS, Kemp AM, Theobald PS, Maguire SA, Jones MD (2013) Do chest compressions during simulated infant CPR comply with international recommendations? Arch Dis Child 98:576-581

229. Martin P, Theobald P, Kemp A, Maguire S, Maconochie I, Jones M (2013) Real-time feedback can improve infant manikin cardiopulmonary resuscitation by up to $79 \%$ - a randomised controlled trial. Resuscitation 84:1125-1130

230. Moya F, James LS, Burnard ED, Hanks EC (1962) Cardiac massage in the newborn infant through the intact chest. Am J Obstet Gynecol 84:798803

231. Park J, Yoon C, Lee JC et al (2014) Manikin-integrated digital measuring system for assessment of infant cardiopulmonary resuscitation techniques. IEEE J Biomed Health Inform 18:1659-1667

232. Todres ID, Rogers MC (1975) Methods of external cardiac massage in the newborn infant. J Pediatr 86:781-782

233. Udassi S, Udassi JP, Lamb MA et al (2010) Twothumb technique is superior to two-finger technique during lone rescuer infant manikin CPR. Resuscitation 81:712-717
234. Whitelaw CC, Slywka B, Goldsmith LJ (2000) Comparison of a two-finger versus two-thumb method for chest compressions by healthcare providers in an infant mechanical model. Resuscitation 43:213-216

235. Lim JS, Cho Y, Ryu S et al (2013)Comparison of overlapping (OP) and adjacent thumb positions (AP) for cardiac compressions using the encircling method in infants. Emerg Med J 30:139-142

236. Orlowski JP (1986) Optimum position for external cardiac compression in infants and young children. Ann Emerg Med 15:667-673

237. Phillips GW, Zideman DA (1986) Relation of infant heart to sternum: its significance in cardiopulmonary resuscitation. Lancet 1:1024-1025

238. Saini SS, Gupta N, Kumar P, Bhalla AK, Kaur H (2012) A comparison of two-fingers technique and two-thumbs encircling hands technique of chest compression in neonates. J Perinatol 32:690-694

239. You Y (2009) Optimum location for chest compressions during two-rescuer infant cardiopulmonary resuscitation. Resuscitation $80: 1378-$ 1381

240. Meyer A, Nadkarni V, Pollock A et al (2010) Evaluation of the Neonatal Resuscitation Program's recommended chest compression depth using computerized tomography imaging. Resuscitation 81:544-548

241. Dannevig I, Solevag AL, Saugstad OD, Nakstad B (2012) Lung injury in asphyxiated newborn pigs resuscitated from cardiac arrest - the impact of supplementary oxygen, longer ventilation intervals and chest compressions at different compression-to-ventilation ratios. Open Respir Med 6:89-96

242. Dannevig I, Solevag AL, Sonerud T, Saugstad OD, Nakstad B (2013) Brain inflammation induced by severe asphyxia in newborn pigs and the impact of alternative resuscitation strategies on the newborn central nervous system. Pediatr Res 73:163-170

243. Hemway RJ, Christman C, Perlman J (2013) The $3: 1$ is superior to a $15: 2$ ratio in a newborn manikin model in terms of quality of chest compressions and number of ventilations. Arch Dis Child Fetal Neonatal Ed 98:F42-F45

244. Solevag AL, Dannevig I, Wyckoff M, Saugstad OD Nakstad B (2010) Extended series of cardiac compressions during CPR in a swine model of perinatal asphyxia. Resuscitation 81:1571-1576

245. Solevag AL, Dannevig I, Wyckoff M, Saugstad OD Nakstad B (2011) Return of spontaneous circulation with a compression:ventilation ratio of 15:2 versus 3:1 in newborn pigs with cardiac arrest due to asphyxia. Arch Dis Child Fetal Neonatal Ed 96:F417-F421

246. Solevag AL, Madland JM, Gjaerum E, Nakstad B (2012) Minute ventilation at different compression to ventilation ratios, different ventilation rates, and continuous chest compressions with asynchronous ventilation in a newborn manikin. Scand J Trauma Resusc Emerg Med 20:73

247. Dean JM, Koehler RC, Schleien CL et al (1991) Improved blood flow during prolonged cardiopulmonary resuscitation with $30 \%$ duty cycle in infant pigs. Circulation 84:896-904

248. Berkowitz ID, Chantarojanasiri T, Koehler RC et al (1989) Blood flow during cardiopulmonary resuscitation with simultaneous compression and ventilation in infant pigs. Pediatr Res 26:558-564
249. Linner R, Werner O, Perez-de-Sa V, Cunha-Goncalves $D$ (2009) Circulatory recovery is as fast with air ventilation as with $100 \%$ oxygen after asphyxia-induced cardiac arrest in piglets. Pediatr Res 66:391-394

250. Lipinski CA, Hicks SD, Callaway CW (1999) Normoxic ventilation during resuscitation and outcome from asphyxial cardiac arrest in rats. Resuscitation 42:221-229

251. Perez-de-Sa V, Cunha-Goncalves D, Nordh A et al (2009) High brain tissue oxygen tension during ventilation with $100 \%$ oxygen after fetal asphyxia in newborn sheep. Pediatr Res 65:57-61

252. Solevag AL, Dannevig I, Nakstad B, Saugstad OD (2010) Resuscitation of severely asphyctic newborn pigs with cardiac arrest by using $21 \%$ or $100 \%$ oxygen. Neonatology 98:64-72

253. Temesvari P, Karg E, Bodi I et al (2001) Impaired early neurologic outcome in newborn piglets reoxygenated with $100 \%$ oxygen compared with room air after pneumothorax-induced asphyxia. Pediatr Res 49:812-819

254. Walson KH, Tang M, Glumac A et al (2011) Normoxic versus hyperoxic resuscitation in pediatric asphyxial cardiac arrest: effects on oxidative stress. Crit Care Med 39:335-343

255. Yeh ST, Cawley RJ, Aune SE, Angelos MG (2009) Oxygen requirement during cardiopulmonary resuscitation (CPR) to effect return of spontaneous circulation. Resuscitation 80:951-955

256. Berg RA, Henry C, Otto CW et al (1996) Initial end-tidal $\mathrm{CO} 2$ is markedly elevated during cardiopulmonary resuscitation after asphyxial cardiac arrest. Pediatr Emerg Care 12:245-248

257. Bhende MS, Karasic DG, Menegazzi JJ (1995) Evaluation of an end-tidal $\mathrm{CO} 2$ detector during cardiopulmonary resuscitation in a canine model for pediatric cardiac arrest. Pediatr Emerg Care 11:365-368

258. Bhende MS, Thompson AE (1995) Evaluation of an end-tidal $\mathrm{CO} 2$ detector during pediatric cardiopulmonary resuscitation. Pediatrics 95:395-399

259. Bhende MS, Karasic DG, Karasic RB (1996) End-tidal carbon dioxide changes during cardiopulmonary resuscitation after experimental asphyxial cardiac arrest. Am J Emerg Med 14:349-350

260. Chalak LF, Barber CA, Hynan L, Garcia D, Christie L, Wyckoff MH (2011) End-tidal CO(2) detection of an audible heart rate during neonatal cardiopulmonary resuscitation after asystole in asphyxiated piglets. Pediatr Res 69:401-405

261. Crespo SG, Schoffstall JM, Fuhs LR, Spivey WH (1991) Comparison of two doses of endotracheal epinephrine in a cardiac arrest model. Ann Emerg Med 20:230-234

262. Jasani MS, Nadkarni VM, Finkelstein MS, Mandell GA, Salzman SK, Norman ME (1994) Effects of different techniques of endotracheal epinephrine administration in pediatric porcine hypoxic-hypercarbic cardiopulmonary arrest. Crit Care Med 22:1174-1180

263. Mielke LL, Frank C, Lanzinger MJ et al (1998) Plasma catecholamine levels following tracheal and intravenous epinephrine administration in swine. Resuscitation 36:187-192

264. Roberts JR, Greenberg MI, Knaub MA, Kendrick ZV, Baskin SI (1979) Blood levels following intravenous and endotracheal epinephrine administration. JACEP 8:53-56

265. Hornchen U, Schuttler J, Stoeckel H, Eichelkraut W, Hahn N (1987) Endobronchial instillation of epinephrine during cardiopulmonary resuscitation. Crit Care Med 15:1037-1039 
266. Wyckoff MH, Perlman JM, Laptook AR (2005) Use of volume expansion during delivery room resuscitation in near-term and term infants. Pediatrics 115:950-955

267. Harrington DJ, Redman CW, Moulden M, Greenwood CE (2007) The long-term outcome in surviving infants with Apgar zero at 10 min: a systematic review of the literature and hospital-based cohort. Am J Obstet Gynecol 196:463 e1-5

268. Lee SK, Penner PL, Cox M (1991) Comparison of the attitudes of health care professionals and parents toward active treatment of very low birth weight infants. Pediatrics 88:110-114

269. Kopelman LM, Irons TG, Kopelman AE (1988) Neonatologists judge the „Baby Doe“ regulations. $\mathrm{N}$ Engl J Med 318:677-683

270. Sanders MR, Donohue PK, Oberdorf MA, Rosenkrantz TS, Allen MC (1995) Perceptions of the limit of viability: neonatologists' attitudes toward extremely preterm infants. J Perinatol 15:494502

271. Rysavy MA, Li L, Bell EF et al (2015) Between-hospital variation in treatment and outcomes in extremely preterm infants. N Engl J Med 372:18011811

272. Patel H, Beeby PJ (2004) Resuscitation beyond 10 min of term babies born without signs of life. $J$ Paediatr Child Health 40:136-138

273. Casalaz DM, Marlow N, Speidel BD (1998) Outcome of resuscitation following unexpected apparent stillbirth. Arch Dis Child Fetal Neonatal Ed 78:F112-F115

274. Kasdorf E, Laptook A, Azzopardi D, Jacobs S, Perlman JM (2015) Improving infant outcome with a 10 min Apgar of 0 . Arch Dis Child Fetal Neonatal Ed 100:F102-F105

275. Laptook AR, Shankaran S, Ambalavanan N et al (2009) Outcome of term infants using apgar scores at 10 min following hypoxic-ischemic encephalopathy. Pediatrics 124:1619-1626

276. Sarkar S, Bhagat I, Dechert RE, Barks JD (2010) Predicting death despite therapeutic hypothermia in infants with hypoxic-ischaemic encephalopathy. Arch Dis Child Fetal Neonatal Ed 95:F423-F428

277. Bottoms SF, Paul RH, Mercer BM et al (1999) Obstetric determinants of neonatal survival: antenatal predictors of neonatal survival and morbidity in extremely low birth weight infants. Am J Obstet Gynecol 180:665-669

278. Ambalavanan N, Carlo WA, Bobashev G et al (2005) Prediction of death for extremely low birth weight neonates. Pediatrics 116:1367-1373

279. Manktelow BN, Seaton SE, Field DJ, Draper ES (2013) Population-based estimates of in-unit survival for very preterm infants. Pediatrics 131:e425-e432

280. Medlock S, Ravelli AC, Tamminga P, Mol BW, AbuHanna A (2011) Prediction of mortality in very premature infants: a systematic review of prediction models. PloS one 6:e23441

281. Tyson JE, Parikh NA, Langer J et al (2008) Intensive care for extreme prematurity - moving beyond gestational age. N Engl J Med 358:16721681

282. Marlow N, Bennett C, Draper ES, Hennessy EM, Morgan AS, Costeloe KL (2014) Perinatal outcomes for extremely preterm babies in relation to place of birth in England: the EPICure 2 study. Arch Dis Child Fetal Neonatal Ed 99:F181-F188

283. Nuffield Council on Bioethics (2006) Critical care decisions in fetal and neonatal medicine: ethical issues. ISBN 190438414
284. Swamy R, Mohapatra S, Bythell M, Embleton ND (2010) Survival in infants live born at less than 24 weeks' gestation: the hidden morbidity of nonsurvivors. Arch Dis Child Fetal Neonatal Ed

285. Baskett PJ, Steen PA, Bossaert L (2005) European Resuscitation Council guidelines for resuscitation 2005. Section 8. The ethics of resuscitation and end-of-life decisions. Resuscitation 67(Suppl 1):S171-S180

286. Fulbrook P, Latour J, Albarran J et al (2007) The presence of family members during cardiopulmonary resuscitation: European federation of Critical Care Nursing associations, European Society of Paediatric and Neonatal Intensive Care and European Society of Cardiology Council on Cardiovascular Nursing and Allied Professions Joint Position Statement. Eur J Cardiovasc Nurs 6:255258

287. Brambrink AM, Ichord RN, Martin LJ, Koehler RC Traystman RJ (1999) Poor outcome after hypoxia-ischemia in newborns is associated with physiological abnormalities during early recovery. Possible relevance to secondary brain injury after head trauma in infants. Exp Toxicol Pathol 51:151-162

288. Vannucci RC, Vannucci SJ (1978) Cerebral carbohydrate metabolism during hypoglycemia and anoxia in newborn rats. Ann Neurol 4:73-79

289. Yager JY, Heitjan DF, Towfighi J, Vannucci RC (1992) Effect of insulin-induced and fasting hypoglycemia on perinatal hypoxic-ischemic brain damage. Pediatr Res 31:138-142

290. Salhab WA, Wyckoff MH, Laptook AR, Perlman JM (2004) Initial hypoglycemia and neonatal brain injury in term infants with severe fetal acidemia. Pediatrics 114:361-366

291. Kent TA, Soukup VM, Fabian RH (2001) Heterogeneity affecting outcome from acute stroke therapy: making reperfusion worse. Stroke 32:23182327

292. Srinivasan V, Spinella PC, Drott HR, Roth CL, Helfaer MA, Nadkarni V (2004) Association of timing duration, and intensity of hyperglycemia with intensive care unit mortality in critically ill children. Pediatr Crit Care Med 5:329-336

293. Klein GW, Hojsak JM, Schmeidler J, Rapaport R (2008) Hyperglycemia and outcome in the pediatric intensive care unit. J Pediatr 153:379-384

294. LeBlanc MH, Huang M, Patel D, Smith EE, Devidas $M$ (1994) Glucose given after hypoxic ischemia does not affect brain injury in piglets. Stroke 25:1443-1447. (discussion 8)

295. Hattori H, Wasterlain CG (1990) Posthypoxic glucose supplement reduces hypoxic-ischemic brain damage in the neonatal rat. Ann Neurol 28:122128

296. Edwards AD, Brocklehurst $P$, Gunn AJ et al (2010) Neurological outcomes at 18 months of age after moderate hypothermia for perinatal hypoxic ischaemic encephalopathy: synthesis and metaanalysis of trial data. BMJ 340:c363

297. Gluckman PD, Wyatt JS, Azzopardi D et al (2005) Selective head cooling with mild systemic hypothermia after neonatal encephalopathy: multicentre randomised trial. Lancet 365:663-670

298. Shankaran S, Laptook AR, Ehrenkranz RA et al (2005) Whole-body hypothermia for neonates with hypoxic-ischemic encephalopathy. N Engl J Med 353:1574-1584

299. Azzopardi DV, Strohm B, Edwards AD et al (2009) Moderate hypothermia to treat perinatal asphyxial encephalopathy. N Engl J Med 361:13491358
300. Eicher DJ, Wagner CL, Katikaneni LP et al (2005) Moderate hypothermia in neonatal encephalopathy: efficacy outcomes. Pediatr Neurol 32:1117

301. Azzopardi D, Strohm B, Marlow N et al (2014) Effects of hypothermia for perinatal asphyxia on childhood outcomes. New Engl J Med 371:140 149

302. Iliodromiti S, Mackay DF, Smith GC, Pell JP, Nelson SM (2014) Apgar score and the risk of cause-specific infant mortality: a population-based cohort study. Lancet 384:1749-1755

303. Rudiger M, Braun N, Aranda J et al (2015) Neonatal assessment in the delivery room - Trial to Evaluate a Specified Type of Apgar (TEST-Apgar). BMC Pediatr 15:18

304. Dalili H, Nili F, Sheikh M, Hardani AK, Shariat M, Nayeri F (2015) Comparison of the four proposed Apgar scoring systems in the assessment of birth asphyxia and adverse early neurologic outcomes. PloS One 10:e0122116

305. Savoldelli GL, Naik VN, Park J, Joo HS, Chow R, Hamstra SJ (2006) Value of debriefing during simulated crisis management: oral versus videoassisted oral feedback. Anesthesiology 105:279285

306. Edelson DP, Litzinger B, Arora V et al (2008) Improving in-hospital cardiac arrest process and outcomes with performance debriefing. Arch Intern Med 168:1063-1069

307. DeVita MA, Schaefer J, Lutz J, Wang H, Dongilli T (2005) Improving medical emergency team (MET) performance using a novel curriculum and a computerized human patient simulator. Qual Saf Health Care 14:326-331

308. Wayne DB, Butter J, Siddall VJ et al (2005) Simulation-based training of internal medicine residents in advanced cardiac life support protocols: a randomized trial. Teach Learn Med 17:210-216

309. Clay AS, Que L, Petrusa ER, Sebastian M, Govert $J$ (2007) Debriefing in the intensive care unit: a feedback tool to facilitate bedside teaching. Crit Care Med 35:738-754

310. Blum RH, Raemer DB, Carroll JS, Dufresne RL, Cooper JB (2005) A method for measuring the effectiveness of simulation-based team training for improving communication skills. Anesth Analg 100:1375-1380. (table of contents)

311. Rudiger M, Braun N, Gurth H, Bergert R, Dinger $J$ (2011) Preterm resuscitation I: clinical approaches to improve management in delivery room. Early Hum Dev 87:749-753

312. Schmid MB, Reister F, Mayer B, Hopfner RJ, Fuchs $\mathrm{H}$, Hummler HD (2013) Prospective risk factor monitoring reduces intracranial hemorrhage rates in preterm infants. Dtsch Arztebl Int 110:489496 